\title{
Formulation of an Experimental Substructure Model Using a Craig-Bampton Based Transmission Simulator
}

\author{
Daniel C. Kammer* \\ Mathew S. Allen \\ Department of Engineering Physics \\ University of Wisconsin \\ 1500 Engineering Dr., Madison, WI 53706 \\ kammer@engr.wisc.edu \\ msallen@engr.wisc.edu \\ Randy L. Mayes \\ Structural Dynamics \\ Sandia National Laboratories ${ }^{1}$, Albuquerque, NM \\ rlmayes@sandia.gov
}

* Corresponding author: kammer@engr.wisc.edu (608) 262-5724

\begin{abstract}
Experimental-analytical substructuring is attractive when there is motivation to replace one or more system subcomponents with an experimental model. This experimentally derived substructure can then be coupled to finite element models of the rest of the structure to predict the system response. The Transmission Simulator method couples a fixture to the component of interest during a vibration test in order to improve the experimental model for the component. The transmission simulator is then subtracted from the tested system to produce the experimental component. The method reduces illconditioning by imposing a least squares fit of constraints between substructure modal coordinates to connect substructures, instead of directly connecting physical interface degrees of freedom. This paper presents an alternative means of deriving the experimental substructure model, in which a Craig-Bampton representation of the transmission simulator is created and subtracted from the experimental measurements. The corresponding modal basis of the transmission simulator is described by the fixedinterface modes, rather than free modes that were used in the original approach. These modes do a better job of representing the shape of the transmission simulator as it responds within the experimental system, leading to more accurate results using fewer modes. The new approach is demonstrated using a simple finite element model based example with a redundant interface.
\end{abstract}

Keywords: experimental substructures, substructuring, Craig-Bampton

\footnotetext{
1 Sandia is a multi-program laboratory operated by Sandia Corporation, a Lockheed Martin Company, for the United States Department of Energy's National Nuclear Security Administration under Contract: DE-AC04-94AL85000
} 


\section{Nomenclature}

dof degrees of freedom

I identity matrix

$\mathbf{k}^{\mathrm{B}}$ exact component B stiffness matrix

K stiffness matrix

$\mathbf{m}^{\mathrm{B}}$ exact component B mass matrix

M mass matrix

P orthogonal projector

q fixed interface modal displacement vector

T transformation matrix

TS transmission simulator

u displacement vector

$\phi \quad$ fixed interface modes of transmission simulator

$\Phi$ free interface modes of transmission simulator

$\Psi \quad$ interior partition of constraint modes

\section{superscripts}
A component A
B component $\mathrm{B}$
C component $\mathrm{C}$
D component D
E system E
$\mathrm{T}$ matrix transpose

subscripts

c interface dof

C constrained interface

CB Craig-Bampton

F Frobenius norm

G uncoupled system

IP interface preserving method

m measurement dof

$\mathrm{m}_{\mathbf{B}}$ measurement locations on component $\mathrm{B}$

$\mathrm{m} / \mathrm{c} \mathrm{m}$ relative to $\mathrm{c}$ 
o interior dof

q modal dof

$\mathrm{S}$ static reduction to interface dof 


\section{Introduction}

Component mode synthesis (CMS) has been a fundamental tool for the structural analysis of large complex systems for years. Instead of the system being modeled as a whole, it is broken up into components or substructures that are modeled using finite elements, and then reduced to a smaller number of degrees of freedom. The substructure approach is often a necessity due to sheer model size. In addition, individual system components are often constructed by different entities leading to separate substructure models. This is especially true in the aerospace community. Substructuring can be used to couple physical or modal models of subcomponents to create a model for the assembly. References [1] and [2] provide an excellent review of competing methods and their relations. For linear systems, one can also operate on the frequency responses directly to predict the response of the assembled system. The latter approach is called frequency based substructuring, or impedance coupling [1].

In recent years, there has been a renewed interest in combining analytical and experimental based substructure models using CMS. Experimental-analytical substructuring is attractive in many applications where there is motivation to replace one subcomponent with an experimental model. For example, a component may be difficult to model if it contains materials with unknown properties, intricate geometry that would require many elements to approximate, or joints or interfaces with unknown stiffness and damping properties. In these scenarios, one can perform a careful dynamic test of the substructure and extract an experimental-based model. This experimental model can then be coupled to finite element models (FEM) of the rest of the structure to predict the system response.

Over the years, a relatively large number of researchers have studied the feasibility of combining analytical and experimental substructures. For example, Martinez, et al. investigated the coupling of an experimental beam like structure and a shell-payload structure [3]. Urgueira [4] considered the need for the inclusion of residual compliances when free interface substructure modes are used, and the difficulty associated with measuring rotations. Morgan et al $[5,6]$ developed a modified residual flexibility approach based on frequency response measured at the substructure interface. A Craig-Bampton [7] substructure representation was then recovered from the measured experimental results. Researchers have attempted to eliminate the need for interface residuals by mass loading the substructure interface during a free-free vibration test. Karpel and Newman [8] developed the fictitious mass method for mass loading the interface of analytical models of substructures. The experimental version, called the boundary mass method [9], required the attachment of large rigid masses to the substructure interface during the experimental vibration test. Ind [10] considered the problem of testing a delicate substructure by attaching a fixture, testing the combined system, and then removing the effects of the fixture from the measured responses using a variety of techniques. The direct approach to substructure coupling would 
be to then enforce compatibility in the physical connection, or interface, degrees of freedom between substructures. Note that "interface" and "connection" are used interchangeably in this paper. Work in this area has resulted in a measured amount of success, but in most cases, significant difficulties were encountered due to lack of sufficient precision in the measurements, uncertainties, numerical illconditioning, inability to measure rotations, nonphysical results, etc. Large errors in system response can result from small errors in subcomponent models [11].

Recently, a new approach was introduced by Allen, Mayes \& Bergman [12], known as the Transmission Simulator (TS) method. In this approach, the component of interest is tested with a fixture, called a transmission simulator, attached at the interface in order to improve the experimental model for the component. This method reduces ill-conditioning by imposing a least squares fit of a constraint connecting two substructures through a set of generalized coordinates corresponding to deformations of the transmission simulator, rather than connecting the substructures directly through the actual physical interface degrees of freedom. The transmission simulator method captures the compliance and damping of the bolted joints at the interface, provides a means for dealing with continuous, compliant interfaces that cannot easily be reduced to a few connection points, and improves the modal basis of the experimental component by exercising the interface. The disadvantage of this approach is that the transmission simulator must be modeled accurately and its model representation must be subtracted from the experimental model, potentially introducing negative mass or stiffness [13,14]. The method has been used with success in several representative examples [15].

In the authors' past work, the transmission simulator was always modeled with a free interface. More specifically, the modal model for the TS was described by up to six rigid body modes and a collection of elastic modes where all of the connection points were free. In traditional FEM-FEM (or analytical-analytical) substructuring, it has been found that using free-free modes to represent a substructure can produce poor synthesized system results. This is especially the case when flexible substructures are rigidly connected [16]. The free-free substructure mode shapes provide a poor basis for describing the shape of the substructure within the system because the interface is not loaded. The Craig-Bampton (CB) substructure representation [7] has become a popular and efficient approach within the aerospace industry for CMS. In this approach, the corresponding modal basis is described by the fixed-interface modes of a substructure, rather than its free modes. Fixed-interface modes tend to do a better job of representing the shape of the substructure as it responds within the synthesized system results. In the current application, even though the TS substructure is being subtracted, not added to a system, the same concept applies. The fixed-interface modes often better represent the deformation shapes of the transmission simulator when it is attached to the substructure being tested. 
This work presents an alternative TS method for developing an experimentally based substructure model, in which a Craig-Bampton finite element model of the TS is created and subtracted from the experimental measurements. It will be shown that this new experimental substructure formulation can be easily connected to a finite element model, and it provides results for predictions of system response that are superior to the usual free-free TS modal formulation.

\section{Theory}

As suggested, in order to properly exercise the interface of a substructure during a free-free vibration test, the interface is connected to a transmission simulator. Ultimately the transmission simulator must be subtracted in order to have an experimental representation of the desired substructure. A simple beam example, shown in Fig. 1, can be used to illustrate the process. Component $C$ is the system tested to obtain experimental results, component $A$ is the transmission simulator, and component $B$ is the substructure for which an experimental representation is desired. The experimental representation of $B$ is obtained by coupling $C$ and a negative FEM representation of $A$.

The physical displacement vector for the transmission simulator, component $A$, can be partitioned into two sets, the $c$-set, corresponding to the interface degrees of freedom (dof) that connect to component $B$, and the $o$-set, corresponding to the interior dof. The resulting partitioned mass and stiffness matrices can be written as

$$
\mathbf{M}^{\mathrm{A}}=\left\lfloor\begin{array}{ll}
\mathbf{M}_{\mathrm{oo}}^{\mathrm{A}} & \mathbf{M}_{\mathrm{oc}}^{\mathrm{A}} \\
\mathbf{M}_{\mathrm{co}}^{\mathrm{A}} & \mathbf{M}_{\mathrm{cc}}^{\mathrm{A}}
\end{array}\right\rfloor \quad \mathbf{K}^{\mathrm{A}}=\left\lfloor\begin{array}{cc}
\mathbf{K}_{\mathrm{oo}}^{\mathrm{A}} & \mathbf{K}_{\mathrm{oc}}^{\mathrm{A}} \\
\mathbf{K}_{\mathrm{co}}^{\mathrm{A}} & \mathbf{K}_{\mathrm{cc}}^{\mathrm{A}}
\end{array}\right\rfloor
$$

The displacement vector for component $A$ can be transformed into CB [7] coordinates using the relation

$$
\mathbf{u}^{\mathrm{A}}=\left\{\begin{array}{c}
\mathbf{u}_{\mathrm{o}}^{\mathrm{A}} \\
\mathbf{u}_{\mathrm{c}}^{\mathrm{A}}
\end{array}\right\}=\left[\begin{array}{cc}
\phi^{\mathrm{A}} & \boldsymbol{\psi}^{\mathrm{A}} \\
0 & \mathbf{I}_{\mathrm{c}}
\end{array}\right]\left\{\begin{array}{c}
\mathbf{q}^{\mathrm{A}} \\
\mathbf{u}_{\mathrm{c}}^{\mathrm{A}}
\end{array}\right\}=\mathbf{T}_{\mathrm{CB}}^{\mathrm{A}} \mathbf{u}_{\mathrm{CB}}^{\mathrm{A}}
$$

where $\phi^{\mathrm{A}}$ is a matrix of $n_{A}$ fixed-interface modes, $\psi^{\mathrm{A}}=-\left(\mathbf{K}_{\mathrm{oo}}^{\mathrm{A}}\right)^{-1} \mathbf{K}_{\mathrm{oc}}^{\mathrm{A}}, \mathbf{I}_{\mathrm{c}}$ is an identity matrix of order equal to the number of interface dof $n_{c}, \mathbf{q}^{\mathrm{A}}$ is the fixed mode displacement vector, and $\mathbf{u}_{\mathrm{c}}^{\mathrm{A}}$ represents the physical displacement of the interface. The CB representation describes the shape of the transmission simulator in terms of dynamic fixed-interface modes, and static shapes, called constraint 
modes $\Psi^{\mathrm{A}}=\left[\begin{array}{ll}\left(\Psi^{\mathrm{A}}\right)^{\mathrm{T}} & \mathbf{I}_{\mathrm{c}}\end{array}\right]^{\mathrm{T}}$, in which each column gives the static response of component $A$ due to a unit deflection of one of the interface dof holding the others fixed. It is important to note that the constraint modes always span the rigid body modes, therefore, the $\mathrm{CB}$ component representation preserves rigid body motion. The corresponding CB mass and stiffness matrices are then given by

$$
\begin{aligned}
& \mathbf{M}_{\mathrm{CB}}^{\mathrm{A}}=\left(\mathbf{T}_{\mathrm{CB}}^{\mathrm{A}}\right)^{\mathrm{T}} \mathbf{M}^{\mathrm{A}} \mathbf{T}_{\mathrm{CB}}^{\mathrm{A}}=\left[\begin{array}{cc}
\mathbf{I}^{\mathrm{A}} & \mathbf{M}_{\mathrm{qc}}^{\mathrm{A}} \\
\mathbf{M}_{\mathrm{cq}}^{\mathrm{A}} & \mathbf{M}_{\mathrm{S}}^{\mathrm{A}}
\end{array}\right] \\
& \mathbf{K}_{\mathrm{CB}}^{\mathrm{A}}=\left(\mathbf{T}_{\mathrm{CB}}^{\mathrm{A}}\right)^{\mathrm{T}} \mathbf{K}^{\mathrm{A}} \mathbf{T}_{\mathrm{CB}}^{\mathrm{A}}=\left[\begin{array}{cc}
\left(\boldsymbol{\omega}^{\mathrm{A}}\right)^{2} & 0 \\
0 & \mathbf{K}_{\mathrm{S}}^{\mathrm{A}}
\end{array}\right]
\end{aligned}
$$

where it has been assumed that the fixed-interface modes have been mass normalized, $\omega^{\mathrm{A}}$ is a diagonal matrix of fixed-interface modal frequencies, and $\mathbf{M}_{\mathrm{S}}^{\mathrm{A}}$ and $\mathbf{K}_{\mathrm{S}}^{\mathrm{A}}$ are the component $A$ mass and stiffness matrices statically reduced to the interface dof, respectively. Note that the stiffness matrix is uncoupled in $\mathrm{CB}$ coordinates, but mass is not. As mentioned previously, the advantage of this transmission simulator representation over a free-free modal representation is that the fixed-interface modes better represent the shape of the simulator in the coupled system being tested.

The CB representation of component $A$ must now be subtracted from the experimental modal representation of the combined system $C$. The uncoupled equation of motion is given by

$$
\left[\begin{array}{cc}
\mathbf{I}^{\mathrm{C}} & 0 \\
0 & -\mathbf{M}_{\mathrm{CB}}^{\mathrm{A}}
\end{array}\right]\left\{\begin{array}{c}
\ddot{\mathbf{q}}^{\mathrm{C}} \\
\ddot{\mathbf{u}}_{\mathrm{CB}}^{\mathrm{A}}
\end{array}\right\}+\left[\begin{array}{cc}
\left(\boldsymbol{\omega}^{\mathrm{C}}\right)^{2} & 0 \\
0 & -\mathbf{K}_{\mathrm{CB}}^{\mathrm{A}}
\end{array}\right]\left\{\begin{array}{c}
\mathbf{q}^{\mathrm{C}} \\
\mathbf{u}_{\mathrm{CB}}^{\mathrm{A}}
\end{array}\right\}=0
$$

where $\mathbf{q}^{\mathrm{C}}$ is the displacement vector for the experimental modes, $\mathbf{I}^{\mathrm{C}}$ is an identity matrix with dimension corresponding to the number of experimental modes retained, $n_{C}$, and $\omega^{\mathrm{C}}$ is a diagonal matrix of the experimental system $C$ frequencies. It is assumed that the response of component $C$ is measured at $n_{m}$ locations on the transmission simulator during the vibration test. In order to couple component $C$ and negative component $A$, the usual approach is to enforce compatible displacements at 
the interface degrees of freedom. However, in general, it is difficult to place sensors and measure all of the connecting degrees of freedom, especially if they are rotations. Following the TS approach [12], the next best coupling method is to enforce physical displacement compatibility between the two components at the measured locations

$$
\mathbf{u}_{\mathrm{m}}^{\mathrm{A}} \quad \mathbf{u}_{\mathrm{m}}^{\mathrm{C}}=0
$$

where the subscript $m$ indicates a row partition corresponding to the measured dof, and it is assumed that none of the interface dof are in the set of measured locations. This paper will discuss two different methods of enforcing the constraint in Eq. (6) using a CB representation for the transmission simulator. This results in two different, but mathematically equivalent representations for the experimental component $B$.

\subsection{Constraint Enforcement using Fixed-Interface TS Modes (CB-IP)}

This approach has been dubbed the CB-IP, or "Interface Preserving", method because the immediate result is a substructure representation for $B$ that still contains the physical interface dof within the displacement vector so they can be directly connected to another FEM. The method proceeds by transforming Eq. (6) to $\mathrm{CB}$ coordinates for component $A$, using Eq. (2), and experimental modal coordinates for component $C$ producing

$$
\phi_{\mathrm{m}}^{\mathrm{A}} \mathbf{q}^{\mathrm{A}}+\psi_{\mathrm{m}}^{\mathrm{A}} \mathbf{u}_{\mathrm{c}}^{\mathrm{A}}-\phi_{\mathrm{m}}^{\mathrm{C}} \mathbf{q}^{\mathrm{C}}=0
$$

Equation (7) can be solved for the transmission simulator fixed-interface modal response

$$
\mathbf{q}^{\mathrm{A}}=\phi_{\mathrm{m}}^{\mathrm{A} \dagger}\left[\phi_{\mathrm{m}}^{\mathrm{C}} \mathbf{q}^{\mathrm{C}}-\psi_{\mathrm{m}}^{\mathrm{A}} \mathbf{u}_{\mathrm{c}}^{\mathrm{A}}\right]=\phi_{\mathrm{m}}^{\mathrm{A} \dagger} \mathbf{u}_{\mathrm{m} / \mathrm{c}}^{\mathrm{C}}
$$

where $\phi_{\mathrm{m}}^{\mathrm{A} \dagger}$ is the Moore-Penrose generalized inverse of the fixed-interface modes row partitioned to the measurement locations. The best estimate of $\mathbf{q}^{\mathrm{A}}$ is obtained when the rank of $\phi_{\mathrm{m}}^{\mathrm{A}}$ is maximized, meaning it becomes full column rank, implying that $n_{m}$ must be greater than $n_{A}$. The term within the bracket

$$
\mathbf{u}_{\mathrm{m} / \mathrm{c}}^{\mathrm{C}}=\phi_{\mathrm{m}}^{\mathrm{C}} \mathbf{q}^{\mathrm{C}}-\psi_{\mathrm{m}}^{\mathrm{A}} \mathbf{u}_{\mathrm{c}}^{\mathrm{A}}
$$


represents the difference between the free response of the measured locations on component $A$ during the vibration test of the combined structure $C$, and the static response of the same measured locations due to unit deflections of the interface dof predicted using the FEM representation of the simulator. It can therefore be considered as an estimate of the response of the measured locations relative to the interface or connection dof.

Premultiplying Eq.(8) by the component $A$ fixed-interface modes at the measurement locations gives

$$
\mathbf{u}_{\mathrm{m} / \mathrm{c}}^{\mathrm{A}}=\phi_{\mathrm{m}}^{\mathrm{A}} \mathbf{q}^{\mathrm{A}}=\phi_{\mathrm{m}}^{\mathrm{A}} \phi_{\mathrm{m}}^{\mathrm{A} \dagger} \mathbf{u}_{\mathrm{m} / \mathrm{c}}^{\mathrm{C}}=\mathbf{P}_{\mathrm{m}}^{\mathrm{A}} \mathbf{u}_{\mathrm{m} / \mathrm{c}}^{\mathrm{C}}=\hat{\mathbf{u}}_{\mathrm{m} / \mathrm{c}}^{\mathrm{C}}
$$

where $\mathbf{P}_{\mathrm{m}}^{\mathrm{A}}$ is an orthogonal projector onto the column space of $\phi_{\mathrm{m}}^{\mathrm{A}}$, and $\hat{\mathbf{u}}_{\mathrm{m} / \mathrm{c}}^{\mathrm{C}}$ is then the orthogonal projection of the response of substructure $C$ at the measurement locations relative to the interface onto this space. The modal constraint process used here is analogous to that used in the usual TS approach, but now it deals with motion of the transmission simulator relative to the interface dof, instead of their total response. The modal constraint approach does not strictly enforce the constraint in Eq. (6), but instead enforces the least-squares fit given in Eq. (10).

The modal constraint is applied by defining the coordinate transformation

$$
\mathbf{u}_{\mathrm{G}}=\left\{\begin{array}{c}
\mathbf{q}^{\mathrm{C}} \\
\mathbf{u}_{\mathrm{CB}}^{\mathrm{A}}
\end{array}\right\}=\left\{\begin{array}{c}
\mathbf{q}^{\mathrm{C}} \\
\mathbf{q}^{\mathrm{A}} \\
\mathbf{u}_{\mathrm{c}}^{\mathrm{A}}
\end{array}\right\}=\left[\begin{array}{cc}
\mathbf{I} & 0 \\
\boldsymbol{\phi}_{\mathrm{m}}^{\mathrm{A}} \boldsymbol{\phi}_{\mathrm{m}}^{\mathrm{C}} & -\boldsymbol{\phi}_{\mathrm{m}}^{\mathrm{A} \dagger} \boldsymbol{\psi}_{\mathrm{m}}^{\mathrm{A}} \\
0 & \mathbf{I}
\end{array}\right]\left\{\begin{array}{c}
\mathbf{q}^{\mathrm{C}} \\
\mathbf{u}_{\mathrm{c}}^{\mathrm{A}}
\end{array}\right\}=\mathbf{T}_{\mathrm{IP}} \mathbf{u}_{\mathrm{r}}
$$

where $\mathbf{u}_{\mathrm{G}}$ is the expanded version of the uncoupled system displacement vector from Eq. (5). Applying the transformation to the uncoupled system in Eq. (5) gives the approximation of the unforced equation of motion for the experimental based substructure $B$

$$
\hat{\mathbf{M}}_{\mathrm{IP}}^{\mathrm{B}} \ddot{\hat{\mathbf{u}}}_{\mathrm{IP}}^{\mathrm{B}}+\hat{\mathbf{K}}_{\mathrm{IP}}^{\mathrm{B}} \hat{\mathbf{u}}_{\mathrm{IP}}^{\mathrm{B}}=0
$$

in which the reduced displacement vector $\mathbf{u}_{\mathrm{r}}$ in Eq. (11) has been renamed $\hat{\mathbf{u}}_{\mathrm{IP}}^{\mathrm{B}}$ and 


$$
\begin{gathered}
\hat{\mathbf{M}}_{\mathrm{IP}}^{\mathrm{B}}=\mathbf{T}_{\mathrm{IP}}^{\mathrm{T}}\left[\begin{array}{cc}
\mathbf{I}^{\mathrm{C}} & 0 \\
0 & -\mathbf{M}_{\mathrm{CB}}^{\mathrm{A}}
\end{array}\right] \mathbf{T}_{\mathrm{IP}}=\left[\begin{array}{cc}
\mathbf{I}^{\mathrm{C}}-\boldsymbol{\tau}_{\mathrm{IP}}^{\mathrm{T}} \boldsymbol{\tau}_{\mathrm{IP}} & \boldsymbol{\tau}_{\mathrm{IP}}^{\mathrm{T}} \boldsymbol{\delta}_{\mathrm{IP}}-\boldsymbol{\tau}_{\mathrm{IP}}^{\mathrm{T}} \mathbf{M}_{\mathrm{qc}}^{\mathrm{A}} \\
\boldsymbol{\delta}_{\mathrm{IP}}^{\mathrm{T}} \boldsymbol{\tau}_{\mathrm{IP}}-\mathbf{M}_{\mathrm{cq}}^{\mathrm{A}} \boldsymbol{\tau}_{\mathrm{IP}} & \mathbf{M}_{\mathrm{cq}}^{\mathrm{A}} \boldsymbol{\delta}_{\mathrm{IP}}+\boldsymbol{\delta}_{\mathrm{IP}}^{\mathrm{T}} \mathbf{M}_{\mathrm{qc}}^{\mathrm{A}}-\boldsymbol{\delta}_{\mathrm{IP}}^{\mathrm{T}} \boldsymbol{\delta}_{\mathrm{IP}}-\mathbf{M}_{\mathrm{s}}^{\mathrm{A}}
\end{array}\right] \\
\hat{\mathbf{K}}_{\mathrm{IP}}^{\mathrm{B}}=\mathbf{T}_{\mathrm{IP}}^{\mathrm{T}}\left[\begin{array}{cc}
\left(\omega^{\mathrm{C}}\right)^{2} & 0 \\
0 & -\mathbf{K}_{\mathrm{CB}}^{\mathrm{A}}
\end{array}\right] \mathbf{T}_{\mathrm{IP}}=\left[\begin{array}{cc}
\left(\boldsymbol{\omega}^{\mathrm{C}}\right)^{2}-\tau_{\mathrm{IP}}^{\mathrm{T}}\left(\boldsymbol{\omega}^{\mathrm{A}}\right)^{2} \tau_{\mathrm{IP}} & \boldsymbol{\tau}_{\mathrm{IP}}^{\mathrm{T}}\left(\boldsymbol{\omega}^{\mathrm{A}}\right)^{2} \boldsymbol{\delta}_{\mathrm{IP}} \\
\boldsymbol{\delta}_{\mathrm{IP}}^{\mathrm{T}}\left(\boldsymbol{\omega}^{\mathrm{A}}\right)^{2} \tau_{\mathrm{IP}} & -\boldsymbol{\delta}_{\mathrm{IP}}^{\mathrm{T}}\left(\boldsymbol{\omega}^{\mathrm{A}}\right)^{2} \boldsymbol{\delta}_{\mathrm{IP}}-\mathbf{K}_{\mathrm{s}}^{\mathrm{A}}
\end{array}\right]
\end{gathered}
$$

with $\tau_{\mathrm{IP}}=\phi_{\mathrm{m}}^{\mathrm{A}^{\dagger}} \phi_{\mathrm{m}}^{\mathrm{C}}$ and $\delta_{\mathrm{IP}}=\phi_{\mathrm{m}}^{\mathrm{A}^{\dagger}} \psi_{\mathrm{m}}^{\mathrm{A}}$. The formulation for component $B$, given by Eqs. (13) and (14), is itself reminiscent of a Craig-Bampton representation, in that the displacement vector $\hat{\mathbf{u}}_{\mathrm{IP}}^{\mathrm{B}}$ contains modal degrees of freedom and the physical degrees of freedom at the points where component $B$ will connect to other substructures. However, it is not precisely a CB form because the stiffness matrix is not block diagonal, so the modal degrees of freedom cannot be truncated based on frequency due to the stiffness coupling between the modal and interface dof. Regardless, the experimental based representation for component $B$ can be easily connected to other FEM based substructures.

\subsubsection{Conservation of Mass and Stiffness}

In general, a substructure's mass matrix must be positive definite, and the stiffness matrix must be positive definite, or semi-definite. However, at this stage, by design, this is not the case in Eqs. (13) and (14) because of the retention of the physical interface dof. There is negative transmission simulator mass and stiffness associated with the interface dof partitions, which make the substructure mass and stiffness matrices for component $B$ indefinite, resulting in nonphysical negative eigenvalues if they were computed at this stage. To make this point more clear, assume that all of the non-interface dof of the transmission simulator are in the measurement set, and all of the transmission simulator fixed-interface modes are retained, then it can be shown that Eqs. (13) and (14) become

$$
\hat{\mathbf{M}}_{\mathrm{IP}}^{\mathrm{B}}=\left[\begin{array}{cc}
\mathbf{I}^{\mathrm{C}}-\phi_{\mathrm{o}}^{\mathrm{CT}} \mathbf{M}_{\mathrm{oo}}^{\mathrm{A}} \phi_{\mathrm{o}}^{\mathrm{C}} & -\phi_{\mathrm{o}}^{\mathrm{CT}} \mathbf{M}_{\mathrm{oc}}^{\mathrm{A}} \\
-\mathbf{M}_{\mathrm{co}}^{\mathrm{A}} \phi_{\mathrm{o}}^{\mathrm{C}} & -\mathbf{M}_{\mathrm{cc}}^{\mathrm{A}}
\end{array}\right]
$$

and

$$
\hat{\mathbf{K}}_{\mathrm{IP}}^{\mathrm{B}}=\left[\begin{array}{cc}
\left(\omega^{\mathrm{C}}\right)^{2}-\phi_{\mathrm{o}}^{\mathrm{CT}} \mathbf{K}_{\mathrm{oo}}^{\mathrm{A}} \phi_{\mathrm{o}}^{\mathrm{C}} & -\phi_{\mathrm{o}}^{\mathrm{CT}} \mathbf{K}_{\mathrm{oc}}^{\mathrm{A}} \\
-\mathbf{K}_{\mathrm{co}}^{\mathrm{A}} \phi_{\mathrm{o}}^{\mathrm{C}} & -\mathbf{K}_{\mathrm{cc}}^{\mathrm{A}}
\end{array}\right]
$$


in which $\phi_{\mathrm{o}}^{\mathrm{C}}$ is the partition of the component $C$ modes corresponding to the non-connection dof of the transmission simulator. The lower right partitions associated with the connection dof of the negative transmission simulator are clearly visible in the matrices for this case. If the connection dof are reduced out of the mass and stiffness matrices in Eqs. (15) and (16) by projecting them onto the component $C$ modal space using $\mathbf{u}_{\mathrm{c}}^{\mathrm{A}}=\phi_{\mathrm{c}}^{\mathrm{C}} \mathbf{q}^{\mathrm{C}}$, then it can be shown that the resulting mass and stiffness matrices become

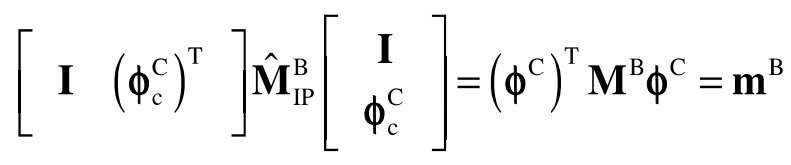

and

$$
\left[\begin{array}{ll}
\mathbf{I} & \left(\phi_{\mathrm{c}}^{\mathrm{C}}\right)^{\mathrm{T}}
\end{array}\right] \hat{\mathbf{K}}_{\mathrm{IP}}^{\mathrm{B}}\left[\begin{array}{c}
\mathbf{I} \\
\phi_{\mathrm{c}}^{\mathrm{C}}
\end{array}\right]=\left(\phi^{\mathrm{C}}\right)^{\mathrm{T}} \mathbf{K}^{\mathrm{B}} \boldsymbol{\phi}^{\mathrm{C}}=\mathbf{k}^{\mathrm{B}}
$$

where $\mathbf{m}^{\mathrm{B}}$ and $\mathbf{k}^{\mathrm{B}}$ are the exact representations of the component $B$ physical mass and stiffness matrices, $\mathbf{M}^{\mathrm{B}}$ and $\mathbf{K}^{\mathrm{B}}$, respectively, in terms of $C$ modal coordinates. This shows that the CB-IP method maintains the correct balance of mass and stiffness in the representation of substructure $B$. In an analogous manner, after directly connecting a CB-IP substructure $B$ to another FEM substructure, the physical connection dof from $B$ can be projected onto the $C$ modal space to eliminate the corresponding negative mass and stiffness contributions.

Another advantage of the CB-IP component $B$ substructure representation is that the fixedinterface modes and frequencies can be determined directly by just crossing out the block row and column associated with the interface dof in Eqs. (13) and (14) yielding

$$
\hat{\mathbf{M}}_{\mathrm{IPC}}^{\mathrm{B}}=\mathbf{I}^{\mathrm{C}}-\tau_{\mathrm{IP}}^{\mathrm{T}} \tau_{\mathrm{IP}} \quad \text { and } \quad \hat{\mathbf{K}}_{\mathrm{IPC}}^{\mathrm{B}}=\left(\boldsymbol{\omega}^{\mathrm{C}}\right)^{2}-\tau_{\mathrm{IP}}^{\mathrm{T}}\left(\boldsymbol{\omega}^{\mathrm{A}}\right)^{2} \tau_{\mathrm{IP}}
$$

Therefore, the fixed-interface modes and frequencies of component $B$ can be estimated based on a freefree vibration test of the composite system $C$ including the transmission simulator. If the proper number of free-free experimental modes for component $C$ and fixed-interface modes from the transmission simulator are retained in the analysis, the matrices $\hat{\mathbf{M}}_{\mathrm{IPC}}^{\mathrm{B}}$ and $\hat{\mathbf{K}}_{\mathrm{IPC}}^{\mathrm{B}}$ should both be positive definite. Techniques presented in Ref. [14] can be used to adjust the fixed component matrices such that they are positive definite, if needed. 


\subsubsection{Theoretical Observations}

Several observations can be made regarding the theory presented for the CB-IP experimental substructure representation. First, the experimental mode set, $\phi^{\mathrm{C}}$, is determined by the frequency range that is desired for experimental component $B$, and the response of $B$ within the selected mode set. Accurate representation of the transmission simulator over the experimental frequency range dictates the fixed-interface mode set $\phi^{\mathrm{A}}$, containing $n_{A}$ modes. As mentioned previously, the motion of $C$ at the measurement locations relative to the motion of the interface must be accurately described by the component $A$ fixed-interface modes, such that the constraint in Eq. (9) is adequately satisfied. Though not directly enforced, the ultimate goal of an accurate subtraction of the transmission simulator from component $C$ is to have the two sets of connection dof be equal, $\mathbf{u}_{\mathrm{c}}^{\mathrm{A}}=\mathbf{u}_{\mathrm{c}}^{\mathrm{C}}=\phi_{\mathrm{c}}^{\mathrm{C}} \mathbf{q}^{\mathrm{C}}$. Substituting this relation into the right most term in the constraint in Eq. (9) gives

$$
\mathbf{u}_{\mathrm{m} / \mathrm{c}}^{\mathrm{A}}=\phi_{\mathrm{m}}^{\mathrm{A}} \mathbf{q}^{\mathrm{A}}=\left[\phi_{\mathrm{m}}^{\mathrm{C}}-\boldsymbol{\psi}_{\mathrm{m}}^{\mathrm{A}} \phi_{\mathrm{c}}^{\mathrm{C}}\right] \mathbf{q}^{\mathrm{C}}=\mathbf{Q} \mathbf{q}^{\mathrm{C}}
$$

Therefore, in order to have an accurate CB-IP formulation for component $B$, the columns in matrix $\mathbf{Q}$ must lie in the range space of the fixed-interface modes row partitioned to the measurement set

$$
\mathbf{Q} \subseteq \mathbf{R}\left(\phi_{\mathrm{m}}^{\mathrm{A}}\right)
$$

The condition in Eq. (21) cannot be checked directly because the connection partition of the component $C$ modes, $\phi_{\mathrm{c}}^{\mathrm{C}}$, cannot be measured during the vibration test. However, an estimate of the required modal partition can be made. In terms of $\mathrm{CB}$ coordinates for the transmission simulator, the constraint in Eq. (6) can be written as

$$
\mathbf{u}_{\mathrm{m}}^{\mathrm{A}}=\mathbf{T}_{\mathrm{CBm}}^{\mathrm{A}} \mathbf{u}_{\mathrm{CB}}^{\mathrm{A}}=\phi_{\mathrm{m}}^{\mathrm{C}} \mathbf{q}^{\mathrm{C}}=\mathbf{u}_{\mathrm{m}}^{\mathrm{C}}
$$

in which $\mathrm{T}_{\mathrm{CBm}}^{\mathrm{A}}$ is the component $A \mathrm{CB}$ transformation matrix row partitioned to the measurement locations, and $\mathbf{u}_{\mathrm{CB}}^{\mathrm{A}}$ is defined in Eq. (2). Solving for $\mathbf{u}_{\mathrm{CB}}^{\mathrm{A}}$ gives

$$
\mathbf{u}_{\mathrm{CB}}^{\mathrm{A}}=\mathbf{T}_{\mathrm{CBm}}^{\mathrm{A} \dagger} \phi_{\mathrm{m}}^{\mathrm{C}} \mathbf{q}^{\mathrm{C}}
$$


Based on the formulation of Eq. (2), the lower partition of Eq. (23) corresponds to the displacement of the connection dof, $\mathbf{u}_{\mathrm{c}}^{\mathrm{A}}$. Assuming that the constraints are all properly applied, the interface displacements of components $A$ and $C$ should be equivalent, therefore an estimate of the interface partition of the retained component $C$ modes is given by

$$
\hat{\phi}_{\mathrm{c}}^{\mathrm{C}}=\left[\mathbf{T}_{\mathrm{CBm}}^{\mathrm{A \dagger}}\right]_{\mathrm{c}} \phi_{\mathrm{m}}^{\mathrm{C}}
$$

This estimate can be substituted into Eq. (20) to check the condition in Eq. (21). Once the CB-IP representation for component $B$ is connected to another FEM component, the physical interface dof from component $B$ can be reduced out of the system matrices by projecting them onto the component $C$ modal coordinates using the estimate in Eq. (24). Note, that to maximize the information contained in $\mathbf{T}_{\mathrm{CBm}}^{\mathrm{A}}$, the measurement locations should be selected such that the columns are linearly independent. This will also ensure a maximum retention of information for the generalized inverse in Eq. (8) because the columns in $\mathbf{T}_{\mathrm{CBm}}^{\mathrm{A}}$ contain the fixed interface modes $\phi_{\mathrm{m}}^{\mathrm{A}}$.

\subsection{Constraint Enforcement using Free-Interface TS Modes (CB-TS)}

This approach is designated the CB-TS method because free-interface shapes of the CB representation of the transmission simulator $A$ are used to enforce the constraint in Eq. (6). Two options are available. Either all of the free-interface modes of the transmission simulator in physical coordinates $\Phi_{\mathrm{CB}}^{\mathrm{A}}$, or all of the columns of the $\mathrm{CB}$ transformation $\mathbf{T}_{\mathrm{CB}}^{\mathrm{A}}$, can be employed in the constraint to subtract the TS from component $C$. For simplicity, the sequel will only consider the use of $\mathbf{T}_{\mathrm{CB}}^{\mathrm{A}}$ directly, but the process is the same using free-interface modes, $\Phi_{\mathrm{CB}}^{\mathrm{A}}$.

The constraint in Eq. (23) can be rewritten as

$$
\mathbf{u}_{\mathrm{CB}}^{\mathrm{A}}=\mathbf{T}_{\mathrm{CBm}}^{\mathrm{A} \dagger} \phi_{\mathrm{m}}^{\mathrm{C}} \mathbf{q}^{\mathrm{C}}=\tau_{\mathrm{TS}} \mathbf{q}^{\mathrm{C}}
$$

and directly applied to the uncoupled equation of motion in Eq. (5) by defining the coordinate transformation 


$$
\mathbf{u}_{\mathrm{G}}=\left\{\begin{array}{c}
\mathbf{q}^{\mathrm{C}} \\
\mathbf{u}_{\mathrm{CB}}^{\mathrm{A}}
\end{array}\right\}=\mathbf{T}_{\mathrm{TS}} \mathbf{q}^{\mathrm{C}}=\left[\begin{array}{c}
\mathbf{I} \\
\tau_{\mathrm{TS}}
\end{array}\right] \mathbf{q}^{\mathrm{C}}
$$

This yields the experimental based representation for the component $B$ mass and stiffness matrices in $C$ modal coordinates as

$$
\hat{\mathbf{M}}_{\mathrm{TS}}^{\mathrm{B}}=\mathbf{T}_{\mathrm{TS}}^{\mathrm{T}}\left[\begin{array}{cc}
\mathbf{I}^{\mathrm{C}} & 0 \\
0 & -\mathbf{M}_{\mathrm{CB}}^{\mathrm{A}}
\end{array}\right] \mathbf{T}_{\mathrm{TS}}=\mathbf{I}^{\mathrm{C}}-\boldsymbol{\tau}_{\mathrm{TS}}^{\mathrm{T}} \mathbf{M}_{\mathrm{CB}}^{\mathrm{A}} \boldsymbol{\tau}_{\mathrm{TS}}
$$

and

$$
\hat{\mathbf{K}}_{\mathrm{TS}}^{\mathrm{B}}=\mathbf{T}_{\mathrm{TS}}^{\mathrm{T}}\left[\begin{array}{cc}
\left(\omega^{\mathrm{C}}\right)^{2} & 0 \\
0 & -\mathbf{K}_{\mathrm{CB}}^{\mathrm{A}}
\end{array}\right] \mathbf{T}_{\mathrm{TS}}=\left(\omega^{\mathrm{C}}\right)^{2}-\tau_{\mathrm{TS}}^{\mathrm{T}} \mathbf{K}_{\mathrm{CB}}^{\mathrm{A}} \boldsymbol{\tau}_{\mathrm{TS}}
$$

If all component $A$ fixed-interface modes are retained and all non-interface dof on component $A$ are measured, then (27) and (28) reduce to the exact expressions for the component $B$ mass and stiffness matrices in $C$ modal coordinates, $\mathbf{m}^{\mathrm{B}}$ and $\mathbf{k}^{\mathrm{B}}$, in Eqs. (17) and (18). A fixed-interface representation of $B$ can also be derived by expanding the constraint in Eq. (22) and setting $\mathbf{u}_{\mathrm{c}}^{\mathrm{A}}$ equal to zero, yielding

$$
\mathbf{q}^{\mathrm{A}}=\phi_{\mathrm{m}}^{\mathrm{A} \dagger} \phi_{\mathrm{m}}^{\mathrm{C}} \mathbf{q}^{\mathrm{C}}=\tau_{\mathrm{TSC}} \mathbf{q}^{\mathrm{C}}=\tau_{\mathrm{IP}} \mathbf{q}^{\mathrm{C}}
$$

Crossing out rows and columns corresponding to $\mathbf{u}_{\mathrm{c}}^{\mathrm{A}}$ in Eq. (5), and applying the constraint gives the fixed-interface mass and stiffness matrices for component $B$ that are identical to those derived using the CB-IP approach in Eq. (19).

The ultimate goal is to connect the experimental based representation of component $B$ to a FEM representation of another component. In the case of the CB-IP approach, the component $B$ interface dof are available for direct connection to another structure. They are subsequently reduced out at the system level using an estimate of the interface partition of the component $C$ mode shapes. In the case of the CB-TS representation of $B$, the interface dof are not explicitly contained in the displacement vector, so they are not directly available for connection. However, Eq. (25) produces an estimate of the relation between the connection dof for $B$ and the modal dof of component $C$ by taking the row partition that corresponds to the physical connection dof, giving 


$$
\mathbf{u}_{\mathrm{c}}^{\mathrm{B}}=\mathbf{u}_{\mathrm{c}}^{\mathrm{A}}=\left[\mathbf{T}_{\mathrm{CBm}}^{\mathrm{A \dagger}}\right]_{\mathrm{c}} \phi_{\mathrm{m}}^{\mathrm{C}} \mathbf{q}^{\mathrm{C}}=\tau_{\mathrm{TSc}} \mathbf{q}^{\mathrm{C}}
$$

For simplicity, assume that component $B$ is to be connected to a single FEM based component $D$ resulting in system $E$. Assuming that $D$ is in a CB format, the uncoupled system mass and stiffness matrices are given by

$$
\mathbf{M}_{\mathrm{G}}^{\mathrm{E}}=\left\lfloor\begin{array}{cc}
\mathbf{M}_{\mathrm{CB}}^{\mathrm{D}} & 0 \\
0 & \hat{\mathbf{M}}_{\mathrm{TS}}^{\mathrm{B}}
\end{array}\right\rfloor \quad \mathbf{K}_{\mathrm{G}}^{\mathrm{E}}=\left\lfloor\begin{array}{cc}
\mathbf{K}_{\mathrm{CB}}^{\mathrm{D}} & 0 \\
0 & \hat{\mathbf{K}}_{\mathrm{TS}}^{\mathrm{B}}
\end{array}\right\rfloor
$$

The system is coupled by enforcing the constraint $\mathbf{u}_{\mathrm{c}}^{\mathrm{D}}=\mathbf{u}_{\mathrm{c}}^{\mathrm{B}}$, which results in the coordinate transformation

$$
\mathbf{u}_{\mathrm{G}}^{\mathrm{E}}=\left\{\begin{array}{c}
\mathbf{u}_{\mathrm{CB}}^{\mathrm{D}} \\
\mathbf{q}^{\mathrm{C}}
\end{array}\right\}=\left\{\begin{array}{c}
\mathbf{q}^{\mathrm{D}} \\
\mathbf{u}_{\mathrm{c}}^{\mathrm{D}} \\
\mathbf{q}^{\mathrm{C}}
\end{array}\right\}=\left[\begin{array}{cc}
\mathbf{I} & 0 \\
0 & \boldsymbol{\tau}_{\mathrm{TSc}} \\
0 & \mathbf{I}
\end{array}\right]\left\{\begin{array}{c}
\mathbf{q}^{\mathrm{D}} \\
\mathbf{q}^{\mathrm{C}}
\end{array}\right\}=\mathbf{T}^{\mathrm{E}} \mathbf{u}^{\mathrm{E}}
$$

System $E$ mass and stiffness matrices are then computed as

$$
\hat{\mathbf{M}}^{\mathrm{E}}=\mathbf{T}^{\mathrm{ET}} \mathbf{M}_{\mathrm{G}}^{\mathrm{E}} \mathbf{T}^{\mathrm{E}} \quad \hat{\mathbf{K}}^{\mathrm{E}}=\mathbf{T}^{\mathrm{ET}} \mathbf{K}_{\mathrm{G}}^{\mathrm{E}} \mathbf{T}^{\mathrm{E}}
$$

The synthesized system $E$ displacement vector contains only subcomponent modal dof, analogous to a Craig-Chang type formulation [17], but with fixed-interface, and mass and stiffness weighted freeinterface subcomponent modal coordinates. It is important to note that the CB-TS approach to component $B$ representation and subsequent system synthesis is totally equivalent to the two-step CB-IP process, in which component $B$ is derived with interface dof preserved, then directly connected to another FEM, and then finally the interface dof are reduced out. In either case, to maximize information for the computation of required pseudo-inverses, measurement locations on the transmission simulator should be selected to render all of the columns of the component $A \mathrm{CB}$ transformation matrix, $\mathbf{T}_{\mathrm{CB}}^{\mathrm{A}}$, linearly independent. 


\subsection{Reduction of Component $C$ Modal Space}

As mentioned previously, the experimental mode set, $\phi^{\mathrm{C}}$, is determined by the frequency range that is desired for experimental component $B$. However, just because a component $C$ mode has a frequency within the desired range, does not necessarily mean that it should be included in the synthesis of component $B$. For example, there may be modes of $C$ that have no motion in the component $B$ portion. There would only be motion in the connected transmission simulator. Subtraction of the transmission simulator would result in indefinite mass and stiffness matrices for component $B$, leading to nonphysical complex eigenvalues [13]. Clearly, modes of this type should not be included in the analysis. A more likely scenario would be to have a subset of $C$ modes in which the $B$ partition is spanned by a smaller number of independent shapes. This would result in an inflated dimension for the component $B$ mass and stiffness matrices that can result in real or complex superfluous modes within the desired frequency band.

In order to identify and remove this problem, sensors should be placed on component $B$ in addition to the sensor set placed on the transmission simulator, component $A$, during the vibration test of combined system $C$. Enough sensors should be placed on $B$, such that its motion can be detected and differentiated within the dynamically important modes of component $C$ within the frequency band of interest. This is no more onerous than what would normally be done during a regular vibration test of the component $C$. The response of the measurement locations on $B$ can be written as

$$
\mathbf{u}_{\mathrm{m}_{\mathrm{B}}}=\phi_{\mathrm{m}_{\mathrm{B}}}^{\mathrm{C}} \mathbf{q}^{\mathrm{C}}
$$

where $\phi_{\mathrm{m}_{\mathrm{B}}}^{\mathrm{C}}$ are the component $C$ modes row partitioned to the measurement locations on $B$. A singular value decomposition can be performed on $\phi_{\mathrm{m}_{\mathrm{B}}}^{\mathrm{C}}$ giving

$$
\mathbf{u}_{\mathrm{m}_{\mathrm{B}}}=\mathbf{U}_{1} \mathbf{S}_{1} \mathbf{V}_{1}^{\mathrm{T}} \mathbf{q}^{\mathrm{C}}=\mathbf{U}_{1} \mathbf{S}_{1} \hat{\mathbf{q}}
$$

in which $\mathbf{S}_{1}$ is a diagonal matrix that has been truncated to $n_{B}$ significantly nonzero singular values, indicating the number of independent shapes that describe the motion of component $B$ within the set of measured $C$ modes. Due to the fact that the columns of $\mathbf{V}_{1}$ are ortho-normal, the $C$ modal space $\mathbf{q}^{\mathrm{C}}$ can be transformed into the reduced space $\hat{q}$ using 


$$
\mathbf{q}^{\mathrm{C}}=\mathbf{V}_{1} \hat{\mathbf{q}}
$$

Redundant information can be eliminated from the formulation of $B$ and the synthesized system by incorporating the transformation in Eq. (36) for $\mathbf{q}^{\mathrm{C}}$ in the preceding analyses.

\section{Numerical Example}

As an example, a system is considered which could lead to an ill-conditioned substructuring problem. The component $B$ of interest is the $\mathrm{C}$-shaped assembly highlighted with a dash-dot (blue) box in Fig. 2 . In the assembled system of interest, $E$ in Fig. 3, component $B$ is connected to another component $D$, consisting of a C-shaped beam creating a box structure with an additional appendage that is $305 \mathrm{~mm}$ long. As shown, the beams in component $D$ are thicker than those in $B$, having a height of $25 \mathrm{~mm}$ where the beams comprising $B$ have a height of $19 \mathrm{~mm}$. The box in system $E$ is $457 \mathrm{~mm}$ square and all of the beams had a width of $25 \mathrm{~mm}$. A transmission simulator, component $A$ highlighted with the dash-dot (red) box in Fig. 2, was designed to replicate the C-shaped beam in component $D$, although the cross section of the transmission simulator was $19 \mathrm{~mm}$ high by $25 \mathrm{~mm}$ thick, so it is less stiff than the corresponding section in $D$. All of the other dimensions were identical to those in component $D$. The interface between component $A$ and component $B$ consists of two separate arms and hence is reminiscent of the system studied by P. Ind in his PhD thesis [10]. Ind's work showed that a system such as this can exhibit dramatic sensitivity to noise because the measurements are likely to contain very little relative motion of the arms and yet that motion provides the only available information about how the structure will move once the transmission simulator is removed. However, in this case the problem should not be as poorly conditioned as the system studied by Ind because the transmission simulator does allow some motion of the interface dof in its lower modes of vibration.

Linear finite element models were created for each beam, containing $25 \mathrm{~mm}$ long elements, and the resulting nodes shown in Fig. 4. Duplicate nodes were merged when assembling the system. The models were restricted to in-plane motion, two translations and one rotation at each node. In order to simulate a modal test, the modes of the FEA model for assembly $C$ were computed to $2485 \mathrm{~Hz}$., resulting in a 16-mode model for $C$ that included three rigid body modes. The corresponding mode shapes are illustrated in Fig. 5. The higher modes show considerable bending of the frame suggesting that a modal model based on these modes should be able to capture relatively complicated motion of component $B$. A singular value decomposition of the component $B$ portion of the $C$ modes revealed that 
the component $C$ modal space did not need to be reduced using the approach discussed at the end of the previous section.

\subsection{Derivation of Experimental Based Component $B$}

The CB-TS approach was selected to generate the simulated experimental based representation for component $B$, due to its more straightforward application. As mentioned previously, the CB-IP method gives identical results. In order to study the convergence of the solution with respect to the number of retained TS fixed-interface modes, 32 sensors were placed using all of the columns of the transformation matrix, $\mathbf{T}_{\mathrm{CB}}^{\mathrm{A}}$, for a 32 dof $\mathrm{CB}$ representation of the TS. The Effective Independence [18] approach was used to reduce the initial candidate sensor set containing all translational, non-interface TS dof, while maintaining the linear independence of the columns of $\mathbf{T}_{\mathrm{CB}}^{\mathrm{A}}$. This process provides a measurement set on the TS that renders the columns of $\mathbf{T}_{\mathrm{CB}}^{\mathrm{A}}$ linearly independent for $\mathrm{CB}$ representations containing up to 26 fixed-interface modes, along with the 6 constraint modes associated with the interface dof.

The constraint in Eq. (25) results in a free-interface representation of component $B$. It is accurately enforced if the component $C$ modes row-partitioned to the measurement locations form a subspace of the columns of the transmission simulator CB transformation matrix, row-partitioned to the same dof, $\phi_{\mathrm{m}}^{\mathrm{C}} \subset \mathbf{T}_{\mathrm{CBm}}^{\mathrm{A}}$. This criterion can be checked by projecting $\phi_{\mathrm{m}}^{\mathrm{C}}$ onto the orthogonal complement of $\mathbf{T}_{\mathrm{CBm}}^{\mathrm{A}}$ using the orthogonal projection

$$
\mathbf{e}_{\mathrm{f}}=\left[\mathbf{I}-\mathbf{T}_{\mathrm{CBm}}^{\mathrm{A}} \mathbf{T}_{\mathrm{CBm}}^{\mathrm{A} \dagger}\right] \phi_{\mathrm{m}}^{\mathrm{C}}
$$

If $\mathbf{e}_{\mathrm{f}}$ is the null matrix, then the columns of $\mathbf{T}_{\mathrm{CBm}}^{\mathrm{A}}$ span the component $C$ modes at the measurement locations. Figure 6 shows the percentage error in the projection, $100 \times\left(\left\|\mathbf{e}_{\mathrm{f}}\right\|_{\mathbf{F}} /\left\|\phi_{\mathrm{m}}^{\mathrm{C}}\right\|_{\mathbf{F}}\right)$, using the Frobenius norm, as a function of the number of fixed-interface modes retained in the CB representation of the TS. In the case of 10 retained modes, the error is only $0.2 \%$. As the number of retained modes increases, the percentage error decreases until it becomes a computed zero for 26 retained modes.

The constraint in Eq. (29) results in the approximation of a fixed-interface representation of component $B$. This constraint is accurately enforced if enough TS fixed-interface modes are retained so that when row partitioned to the measurement locations, they span the column space of the component $C$ modes at the measurement locations, $\phi_{\mathrm{m}}^{\mathrm{C}} \subset \phi_{\mathrm{m}}^{\mathrm{A}}$. Analogous to the previous computation, this condition 
can be checked by projecting $\phi_{\mathrm{m}}^{\mathrm{C}}$ onto the orthogonal complement of $\phi_{\mathrm{m}}^{\mathrm{A}}$ using the orthogonal projection

$$
\mathbf{e}_{\mathrm{c}}=\left[\mathbf{I}-\phi_{\mathrm{m}}^{\mathrm{A}} \phi_{\mathrm{m}}^{\mathrm{A} \dagger}\right] \phi_{\mathrm{m}}^{\mathrm{C}}
$$

Figure 6 also illustrates the percentage error in the projection for the fixed-interface constraint as a function of the number of fixed-interface modes retained in the CB representation of the TS. It is apparent that the satisfaction of this constraint converges very much more slowly than the previous constraint for the free-free representation of component $B$. This implies that the fixed-interface modes alone do not do a very good job of describing the motion of the TS in the component $C$ modes. In order to achieve an accurate fixed-interface representation for component $B$, more fixed modes must be retained in the $\mathrm{CB}$ formulation of the TS, than is required for the case of an accurate free-free representation.

The quality of the derived experimental based representation of component $B$ can also be examined by comparing its modal frequencies to those predicted by corresponding finite element model. However, it is important to remember that the derived component $B$ is mass and stiffness weighted at the interface. Therefore, it is not strictly appropriate to directly compare all of its frequencies to those of a straightforward free-interface representation of $B$, especially at the higher frequencies. However, the lower frequencies should agree fairly well. In this particular application, for 22 retained fixed-interface modes in the TS, the simulated experimental based component $B$ accurately predicts the three rigid body modes, and all elastic modes below $2400 \mathrm{~Hz}$ agree with the frequencies of the usual free-free representation to within $1.0 \%$ or less. In the case of a fixed-interface representation, a direct comparison can be made. In the same case of 22 retained fixed-interface modes, the corresponding fixed representation of $B$ predicts modal frequencies below $2400 \mathrm{~Hz}$. with up to $5.0 \%$ error. In addition, eight of the 16 predicted frequencies were complex, and thus nonphysical, due to subtraction of noncompatible amounts of TS mass. The indefinite fixed-interface mass matrix can be corrected by applying the approach described in Ref. [14]. In the limit of retaining all of the TS fixed-interface modes and measuring all of the non-interface dof on the TS, the nonphysical modes disappear, and the fixed representation for component $B$ becomes exact. 


\subsection{Synthesis of System $E$}

The true measure of the efficacy of the proposed experimental based component $B$ is the accuracy with which it represents the component within a larger system. The procedure outlined in Eqs. (30) to (33) was used to connect the previously derived experimental component $B$ to the FEM representation of component $D$, shown in Figs. 3 and 4, to form the synthesized system $E$. The accuracy of the synthesized system was determined by comparing its modal parameters with those predicted by the complete system FEM shown in Fig. 4. Note that the synthesized system contains 123 physical noninterface dof in component $D$ and 16 modal dof corresponding to experimental based component $B$. The full system FEM contained 252 physical dof. Figure 7 shows the corresponding FEM system $E$ mode shapes. The FEM and synthesized system modal frequencies are directly compared in Table 1 . The system FEM is assumed to be exact in this example. The synthesized system containing the experimentally based component $B$ accurately predicts the true system frequencies up to $2,650 \mathrm{~Hz}$. This is slightly above the highest frequency of $2,485 \mathrm{~Hz}$ corresponding to the 16 experimental based component $C$ modes used to derive $B$. There are no superfluous or non-physical modes predicted by the synthesized system containing the experimentally derived component $B$. The three rigid body modes are also accurately predicted by the synthesized system. The mode shapes of the synthesized system were transformed back to physical space and compared to the FEM representation of $E$ using a crossorthogonality computation with respect to the full FEM mass matrix given by

$$
\mathbf{C O}=\left(\phi_{\text {SYN }}^{\mathrm{E}}\right)^{\mathrm{T}} \mathbf{M}_{\mathrm{FEM}}^{\mathrm{E}} \phi_{\mathrm{FEM}}^{\mathrm{E}}
$$

The cross-generalized mass (CGM) values (diagonal terms) are also listed in Table 1. All the elastic mode shapes below $2,650 \mathrm{~Hz}$. are accurately predicted by the synthesized system, as indicated by CGM values of 1.00. The corresponding complete $C O$ matrix, shown in Fig. 8, illustrates that there is very little coupling between the synthesized and exact system modes. Therefore, the synthesized system accurately represents the true system modal properties within the relevant frequency range between 0.0 and $2,485 \mathrm{~Hz}$.

The benefit of using a Craig-Bampton representation vs. the usual free-free representation [15] for the transmission simulator in the development of experimental component $B$ can be illustrated by the convergence of the fundamental elastic mode for the synthesized system. In order to have a valid comparison between the two representations, the free-free TS model starts with 6 normal modes, analogous to the 6 static constraint modes in the CB representation of the TS. Therefore, for the first 
iteration, one normal mode would be added to the free-free representation of the TS, and one fixedinterface mode would be added to the $\mathrm{CB}$ representation, producing 7 dof in each case. Figure 9 illustrates the convergence of the fundamental elastic synthesized system frequency as additional modes are added to each TS representation. Clearly, the CB based TS provides superior results for a smaller number of retained modes. The fixed-interface modes of the TS do a superior job of representing the TS within the experimental component $C$, as theorized. Analogous results were observed for higher frequency modes.

\subsection{Robustness of Method}

In the previous analysis, the simulated experimental data for component $C$ was totally consistent with the FEM representation of the TS. In reality, this will never be the case because the FEM based TS will never be a perfect representation. There will always be uncertainty in the TS model. Therefore, in order to examine the robustness of the proposed technique, the FEM mass and stiffness matrices for component $A$ were perturbed using the Maximum Entropy approach developed by Soize [19]. A dispersion level is selected that can be thought of as being analogous to the global fractional uncertainty or error believed to exist in the matrix, and then the matrix is randomized subject to the constraints of maintaining symmetry and positive semi-definiteness. Details of the process can be found in Soize's paper [19]. The advantage of this nonparametric approach, over the usual parameter sensitivity or perturbation methods, is that this randomization process automatically accounts for uncertainties that are not easily described by model parameters, such as model form, geometry, joints, etc. In this example, a $20 \%$ dispersion level was assumed for both the mass and stiffness matrices of the component $A$ transmission simulator. The perturbed TS resulted in a $2.3 \% \mathrm{rms}$ perturbation of the free-free modal frequencies, and a $2.8 \%$ rms perturbation of the corresponding fixed modal frequencies.

The perturbed TS was then used to produce an experimental based component $B$, which was subsequently attached to FEM component $D$ to form system $E$. The number of fixed-interface modes retained in the $\mathrm{CB}$ representation of the perturbed TS was varied to study the convergence of the solution. At 16 retained fixed modes, the predicted frequencies for the first several elastic system modes converged, and beyond 16 fixed modes, the estimates tended to diverge. This behavior makes sense because the TS is not completely accurate. A point is reached at which the accuracy of $B$ no longer improves by adding more fixed-interface modes to the TS. Sixteen retained fixed-interface modes also minimizes the error in the prediction of the component $B$ interface response using Eq. (30). Of course in practice, this information would not be available because response is not measured at the interface. Table 2 lists the predicted system frequencies for the case of 16 retained fixed-interface modes in the 
TS. Even though the perturbed TS used in the analysis does not reflect the reality of experimental component $C$, the largest frequency error for the 13 elastic system modes below the maximum frequency of $2,485 \mathrm{~Hz}$. included in component $C$ is only $1.6 \%$. The corresponding cross-generalized mass values (diagonal terms) are also listed in Table 2. All the elastic mode shapes below 2,485 Hz. are accurately predicted by the synthesized system, as indicated by CGM values between 0.99 and 1.00. The corresponding complete $C O$ matrix, shown in Fig. 10, illustrates that there is still very little coupling between the synthesized and exact system modes. The largest off-diagonal coupling term is 0.16 . Therefore, the synthesized system using the $20 \%$ perturbed TS, still accurately represents the true system modal properties within the relevant frequency range between 0.0 and 2,485 Hz. However, it is important to note that because the TS is not precisely consistent with the experimental TS, there are 4 non-physical system eigenvalues consisting of two negative eigenvalues and one complex conjugate pair. These modes can be easily identified and removed from a system modal model for subsequent system response analysis.

\section{Conclusion}

Experimental-analytical substructuring is attractive when there is motivation to replace one or more system subcomponents with an experimental model. The experimental based substructure can then be coupled to finite element models of the rest of the structure to predict the system response. This paper presented an alternative Transmission Simulator method for developing an experimentally based substructure, in which a Craig-Bampton finite element model of the TS is created and subtracted from the experimental measurements. The corresponding modal basis is described by the fixed-interface modes of the TS, rather than its free modes, as used in the original TS approach. The fixed-interface modes, combined with the corresponding constraint modes, do a better job of representing the shape of the TS as it responds within the experimental system. Two separate formulations, CB-IP and CB-TS, were presented. The CB-IP method retains the physical connection degrees of freedom such that they can be easily attached to another FEM. Subsequently, they must be reduced out using an approximation of the interface motion to remove unwanted negative TS mass and stiffness. This step is avoided in the CB-TS approach, which immediately removes the interface degrees of freedom, but the same interface motion approximation must be used to connect the experimental substructure to another FEM. It was found that both formulations give identical results. The CB-TS formulation seems to be the preferred approach due to its slightly more straightforward application.

The new formulation was applied to a simple example that had a redundant and possibly illconditioned interface. The test data was simulated using a FEM representation to develop the 
experimental based component model, which was subsequently attached to another FEM based component. The resulting system frequencies and mode shapes were compared to those produced by the system FEM. As more fixed-interface modes were retained in the CB-TS representation, the modal parameters of the synthesized system containing the experimental component converged to the exact answers. In addition, it was shown that the new CB-TS approach converges much more rapidly than the original TS method. The new approach was also shown to be robust with respect to differences between the TS FEM representation and the TS that is actually contained in the test article.

Finally, it is important to note that there is currently not an a priori method for determining if a system synthesized by attaching the derived component $B$ to another structure has negative eigenvalues, or inappropriate mass and stiffness matrix sign-definiteness. Instead, the system eigenvalues must be calculated to check their sign. If modal superposition can be used, system modes with nonphysical or negative eigenvalues can be easily removed from the modal model. If direct time integration of the synthesized system matrices is required, then the correct sign-definiteness of the mass matrix can be restored by techniques developed in Ref. [14].

\section{Acknowledgement}

This work was supported by Sandia National Laboratories. Sandia is a multi-program laboratory operated by Sandia Corporation, a Lockheed Martin Company, for the United States Department of Energy’s National Nuclear Security Administration under Contract: DE-AC04-94AL85000

\section{References}

[1] de Klerck, D., D.J. Rixen, and S.N. Voormeeren. "General Framework for Dynamic Substructuring: History, Review, and Classification of Techniques." AIAA Journal, 2008, 46(5), p. 1169-1181.

[2] Herting, D.N., "A General Purpose, Multi-Stage, Component Modal Synthesis Method," Finite Elements in Analysis and Design, 1985, 1(2), p. 153-164.

[3] Martinez, D.R., A.K. Miller, and T.G. Carne, "Combined Experimental and Analytical Modeling of Shell/Payload Structures”, Joint ASCE/ASME Mechanics Conference, 1985, Albuquerque, NM.

[4] Urgueira, A.P.V., "Dynamic Analysis of Coupled Structures Using Experimental Data”, Imperial College of Science, Technology, \& Medicine, 1989, University of London: London.

[5] Morgan, J.A., C. Pierre, and G.M. Hulbert, "Calculation of Component Mode Synthesis Matrices From Measured Frequency Response, Part 1: Theory," Journal of Vibration and Acoustics, 1998, 120(2), p. 503-508. 
[6] Morgan, J.A., C. Pierre, and G.M. Hulbert, "Forced Response of Coupled Substructures Using Experimentally Based Component Mode Synthesis,” AIAA Journal, 1997, 35(2), p. 334339.

[7] Craig, R.R. and M.C.C. Bampton, "Coupling of Substructures for Dynamic Analysis, AIAA Journal, 1968, 6(7), p. 1313-1319.

[8] Karpel, M., and M. Newman, "Accelerated Convergence for Vibration Modes using the Substructure Coupling Method and Fictitious Coupling Masses," Israel Journal of Technology, 1975, 13, p. 55-62.

[9] Karpel, M., and S. Ricci, "Experimental Modal Analysis of Large Structures by Substructuring," Mechanical Systems and Signal Processing, 1997, 11(2), p. 245-256.

[10] Ind, P., "The Non-Intrusive Modal Testing of Delicate and Critical Structures", Imperial College of Science, Technology, \& Medicine. 2004, University of London: London.

[11] Imregun, M., D.A. Robb, and D.J. Ewins, "Structural Modification and Coupling Dynamic Analysis Using Measured FRF Data”, 5th International Modal Analysis Conference, 1987, London, UK.

[12] Allen, M.S., R.L. Mayes, and E.J. Bergman, "Experimental Modal Substructuring to Couple and Uncouple Substructures with Flexible Fixtures and Multi-Point Connections," Journal of Sound and Vibration, 2010, 329(23), p. 4891-4906.

[13] Allen, M.S., D.C. Kammer, and R.L. Mayes, "Metrics for Diagnosing Negative Mass and Stiffness When Uncoupling Experimental and Analytical Substructures," Journal of Sound and Vibration, 2012, 331(25), p. 5435-5448.

[14] Mayes, R.L., M.S. Allen, and D.C. Kammer, "Correcting Indefinite Mass Matrices Due to Substructure Uncoupling," Journal of Sound and Vibration, 2013, 332(22), p. 5856-5866.

[15] Mayes, R.L., and M. Arviso, "Design Studies for the Transmission Simulator Method of Experimental Dynamic Substructuring," International Seminar on Modal Analysis, 2010, Lueven, Belgium.

[16] Baker, M., "Component Mode Synthesis Methods for Test-Based, Rigidly Connected Flexible Components," Journal of Spacecraft and Rockets, 1986, 23(2), p. 316-322.

[17] Craig, R.R., and C-J Chang, "On the Use of Attachment modes in Substructure Coupling for Dynamic Analysis," AIAA/ASME 18th Structures, Structural Dynamics and Materials Conference, 1977, San Diego, CA, p. 89-99.

[18] Kammer, D.C., "Sensor Placement for On-Orbit Modal Identification and Correlation of Large Space Structure", Journal of Guidance, Control, and Dynamics, 1991, 14(2), p. 251-259. 
[19] Soize, C., "Maximum Entropy Approach for Modeling Random Uncertainties in Transient Elastodynamics," Journal of the Acoustic Society of America, 2001, 109(5), p. 1979-1996. 


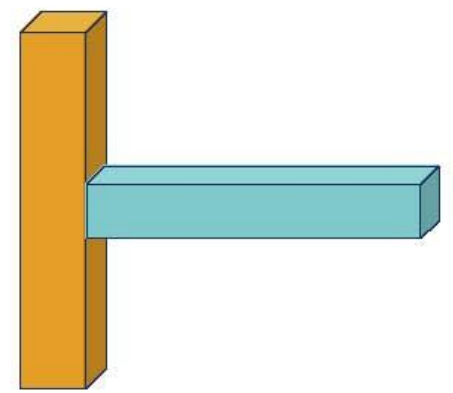

Experimental Component: C

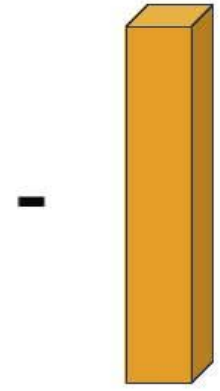

FEM Transmission

Simulator: A

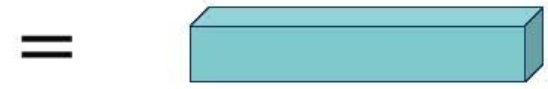

Experimental Based Component: B

Fig. 1. Subtraction of TS from experimental substructure.

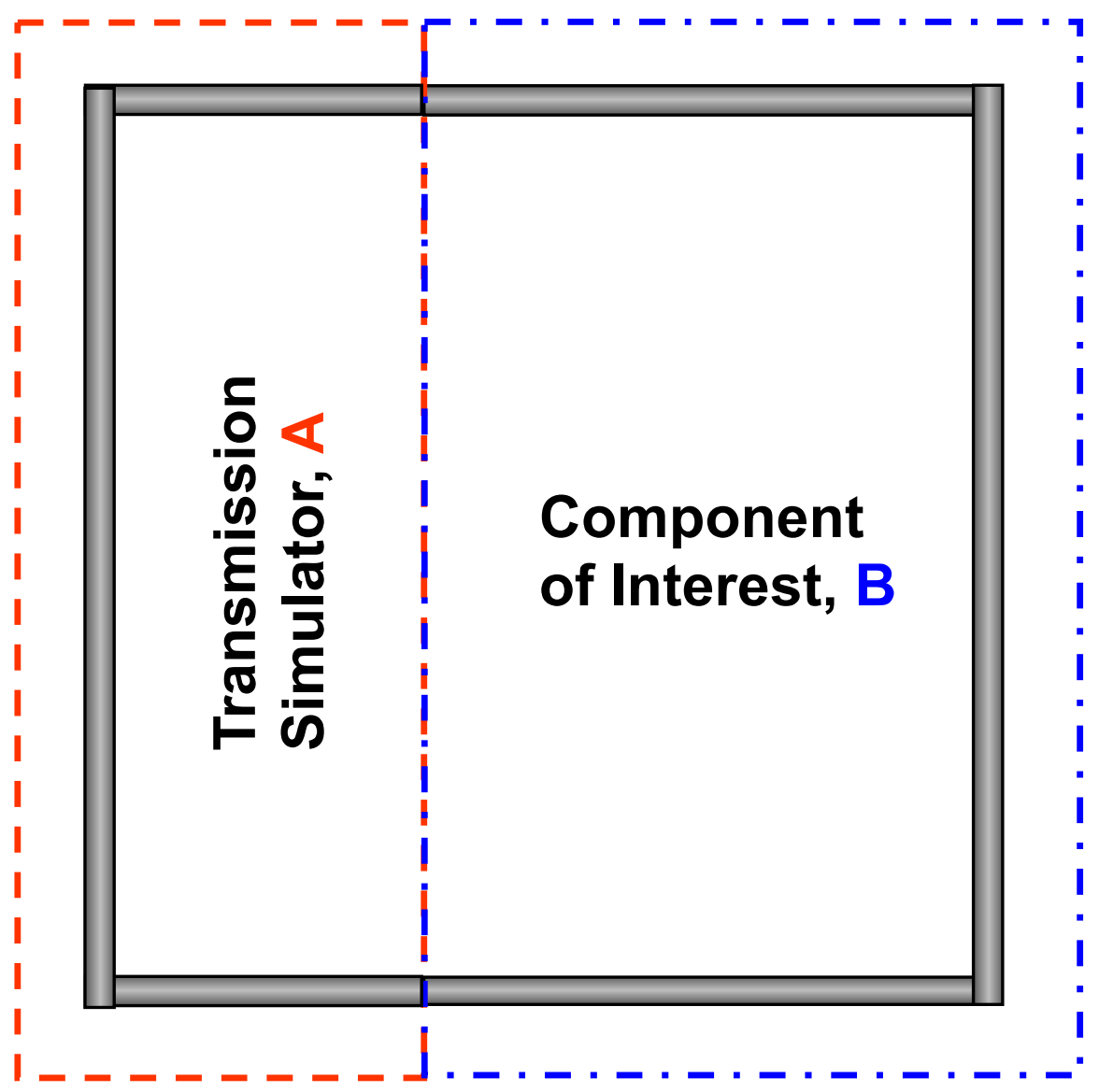

Fig. 2. Schematic of two-dimensional assembly $C$ with a statically indeterminate interface. 


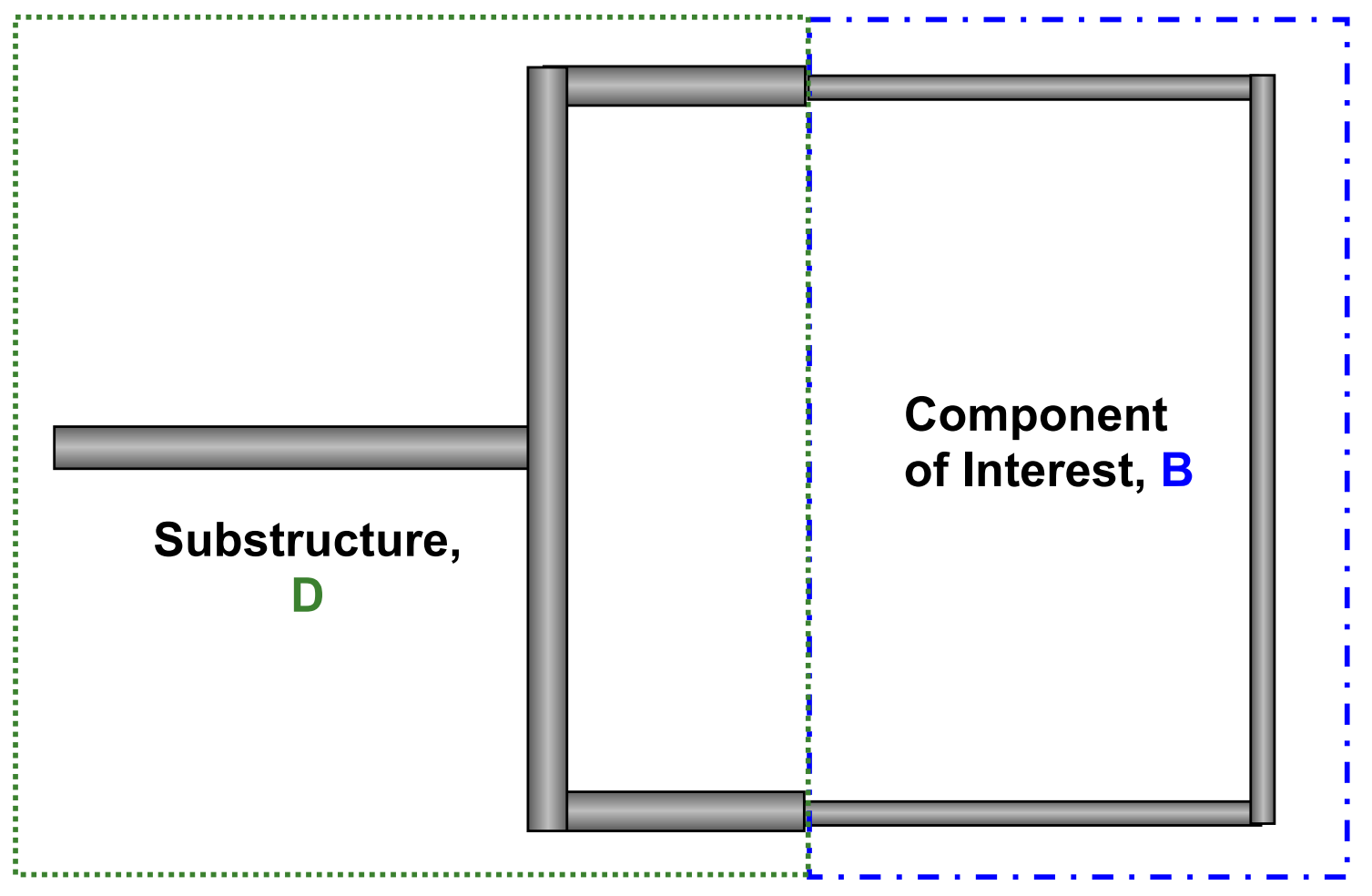

Fig. 3. Full assembly, $E=B+D$.

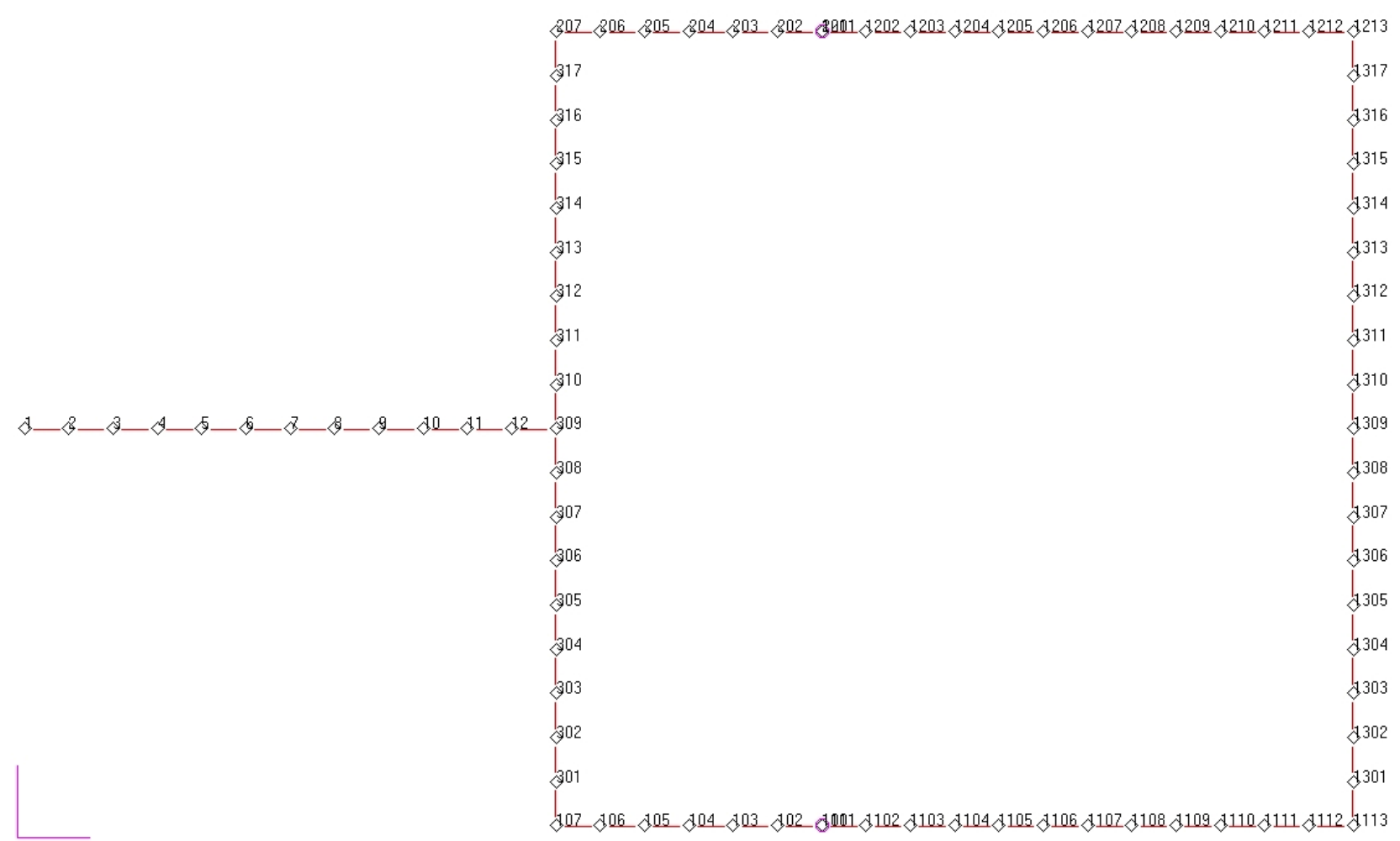

Fig. 4. Finite element mesh of the assembly of interest, $E$. 


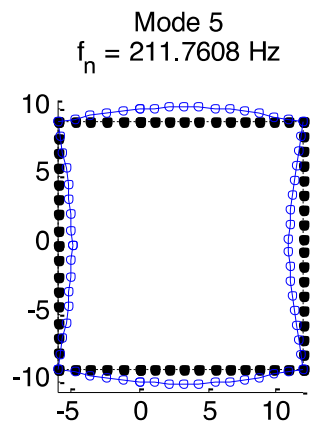

(a)

\section{Mode 9}

$f_{n}=919.5138 \mathrm{~Hz}$

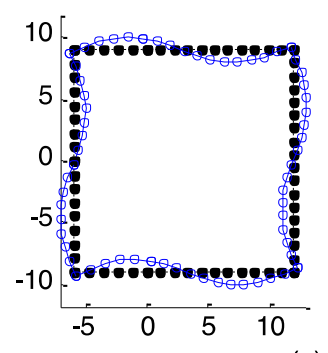

(e)

$$
\begin{gathered}
\text { Mode } 13 \\
f_{n}=1881.4293 \mathrm{~Hz}
\end{gathered}
$$

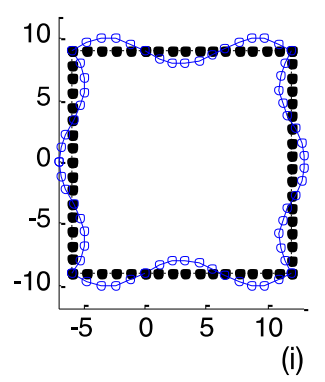

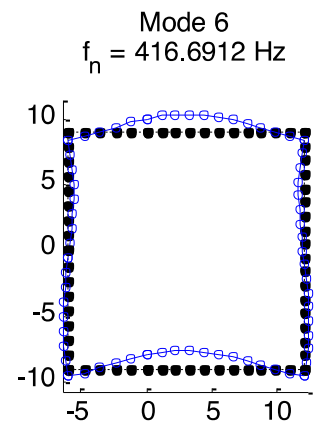

(b)
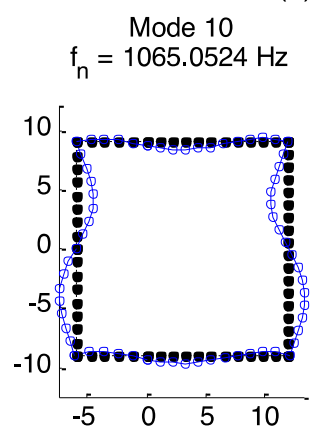

(f)

$$
\begin{aligned}
& \text { Mode } 14 \\
f_{n}= & 2332.9003 \mathrm{~Hz}
\end{aligned}
$$

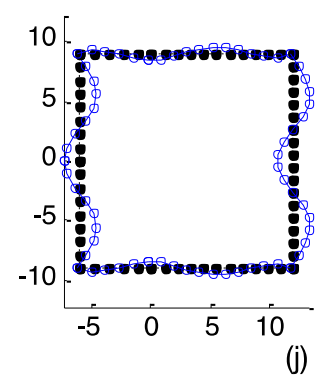

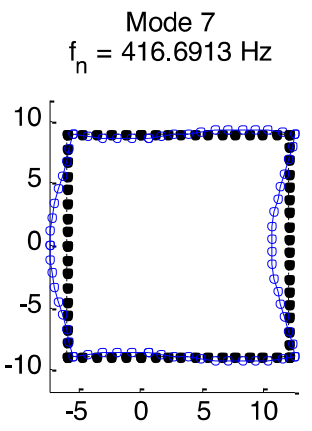

(c)
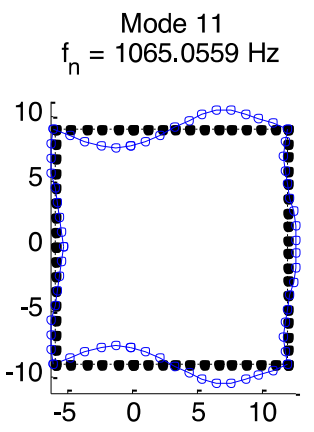

(g)
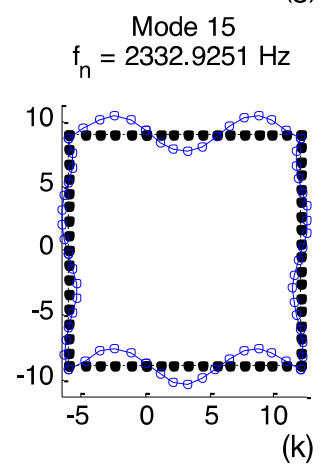

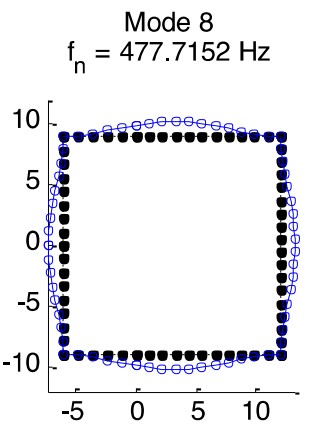

(d)
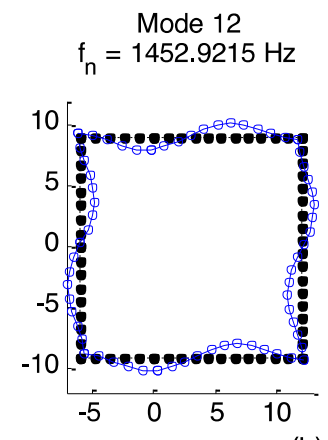

(h) $\begin{aligned} & \text { Mode } 16 \\ f_{n}= & 2485.539 \mathrm{~Hz}\end{aligned}$

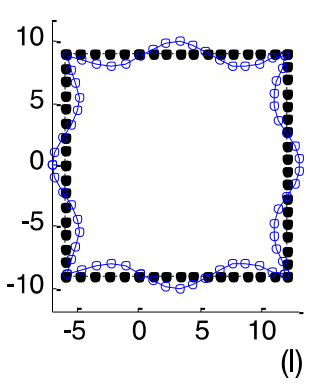

Fig. 5. Component $C$ elastic modes 5 (a) through 16 (l). 


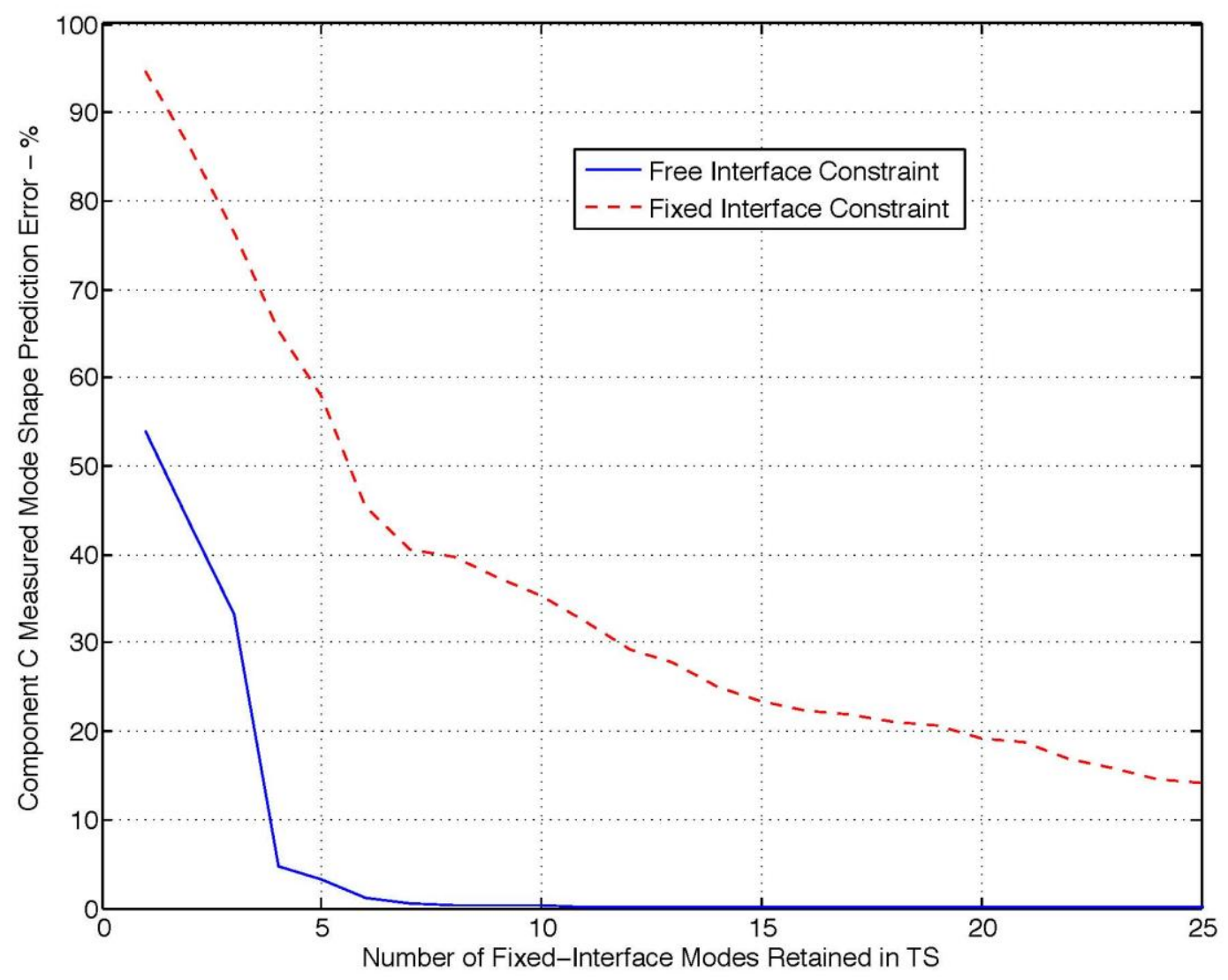

Fig. 6. Percentage error in satisfaction of constraints for free- and fixed-interface component $B$. 


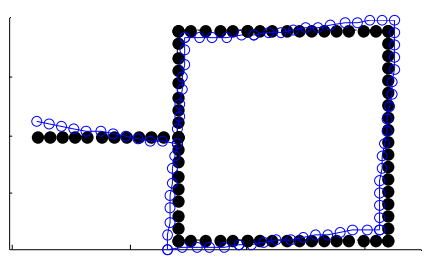

(a)

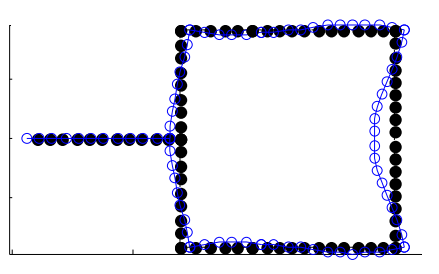

(d)

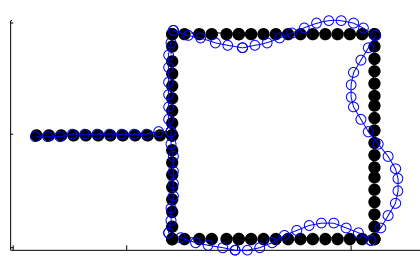

(g)

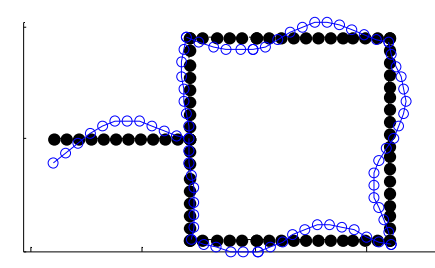

(j)
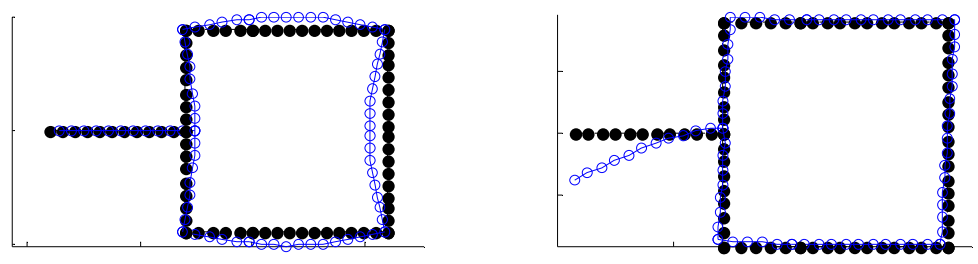

(b)

(c)
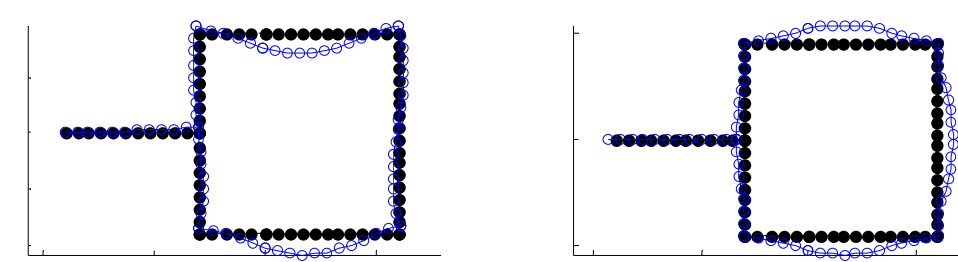

(e)

(f)
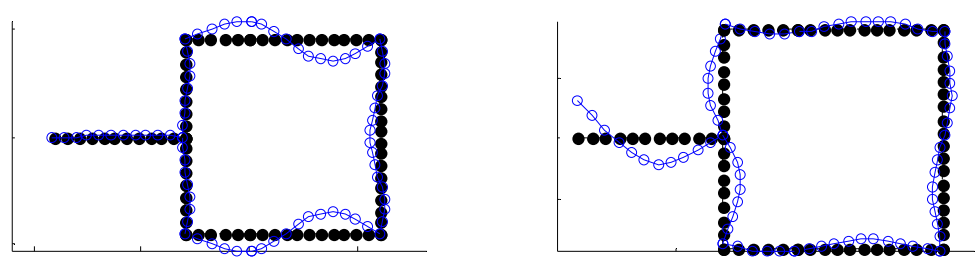

(h)

(i)
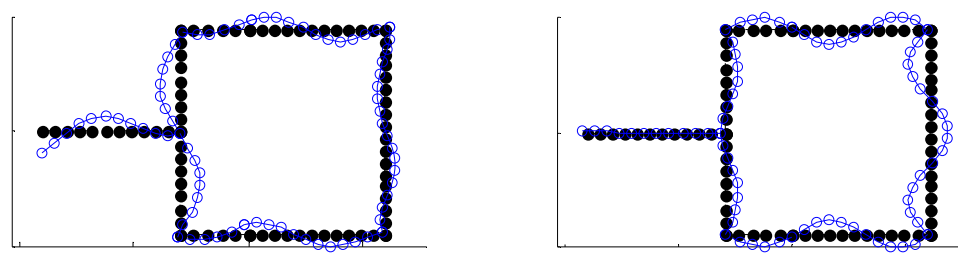

(k)

Fig. 7. Exact elastic mode shapes 4 (a) through 15 (1) for system $E$ FEM representation. 
Table 1. System $E$ modal parameter comparison.

$\begin{array}{rrrrr}\frac{\text { Mode }}{4.00} & \frac{\text { Exact }}{138.99} & \frac{\text { Predicted }}{139.00} & \frac{\text { Error \% }}{-0.01} & \frac{\text { CGM }}{1.00} \\ 5.00 & 207.87 & 207.86 & 0.01 & 1.00 \\ 6.00 & 229.35 & 229.35 & -0.00 & 1.00 \\ 7.00 & 414.35 & 414.36 & -0.00 & 1.00 \\ 8.00 & 456.82 & 456.80 & 0.00 & 1.00 \\ 9.00 & 489.64 & 489.62 & 0.01 & 1.00 \\ 10.00 & 985.64 & 985.43 & 0.02 & 1.00 \\ 11.00 & 1146.41 & 1144.77 & 0.14 & 1.00 \\ 12.00 & 1179.27 & 1179.21 & 0.00 & 1.00 \\ 13.00 & 1355.92 & 1354.96 & 0.07 & 1.00 \\ 14.00 & 1864.29 & 1863.01 & 0.07 & 1.00 \\ 15.00 & 1981.94 & 1980.29 & 0.08 & 1.00 \\ 16.00 & 2411.89 & 2411.55 & 0.01 & 1.00 \\ 17.00 & 2612.74 & 2595.38 & 0.66 & 1.00 \\ 18.00 & 2642.43 & 2629.35 & 0.50 & 1.00\end{array}$

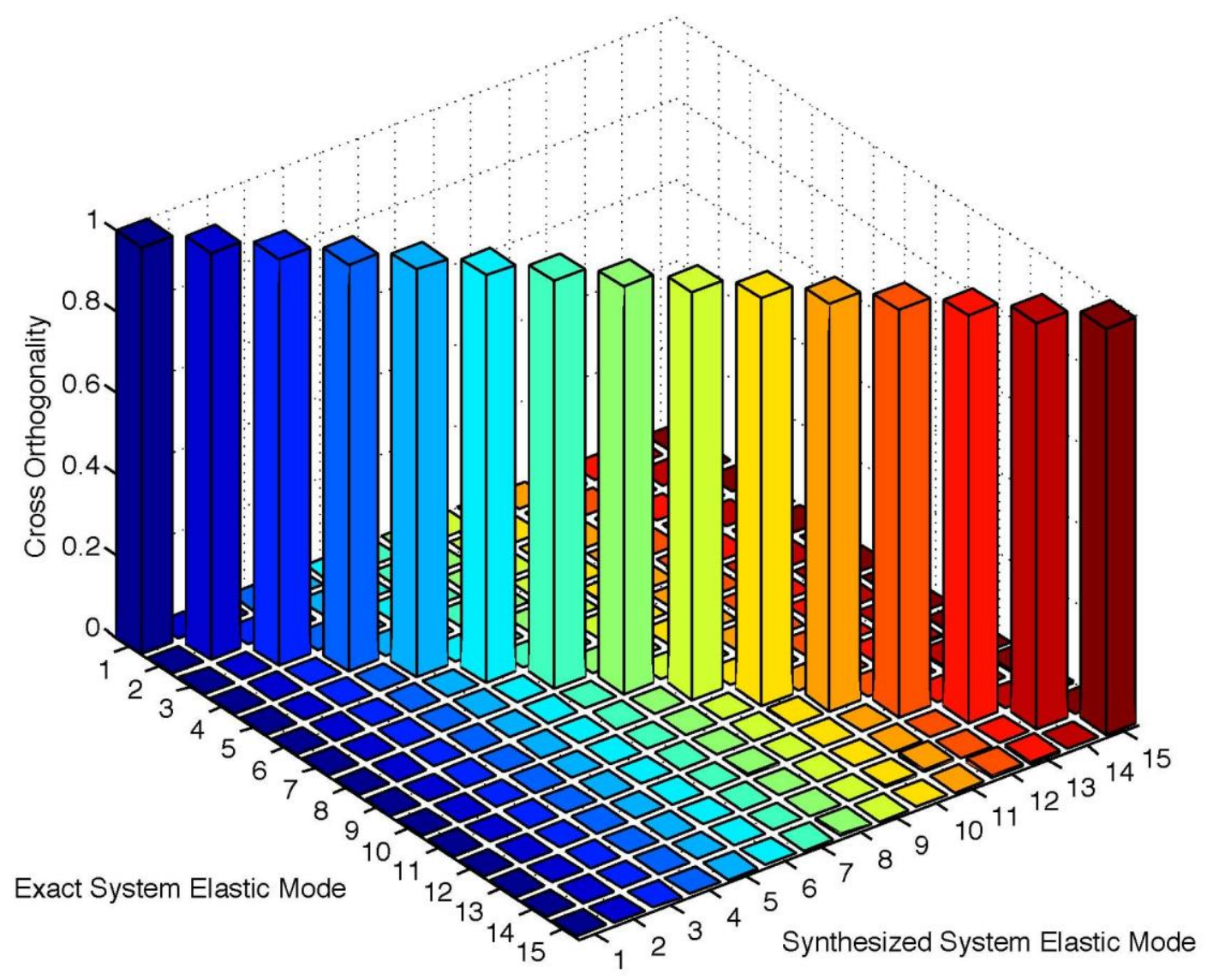

Fig. 8. System cross-orthogonality for first 15 elastic modes. 


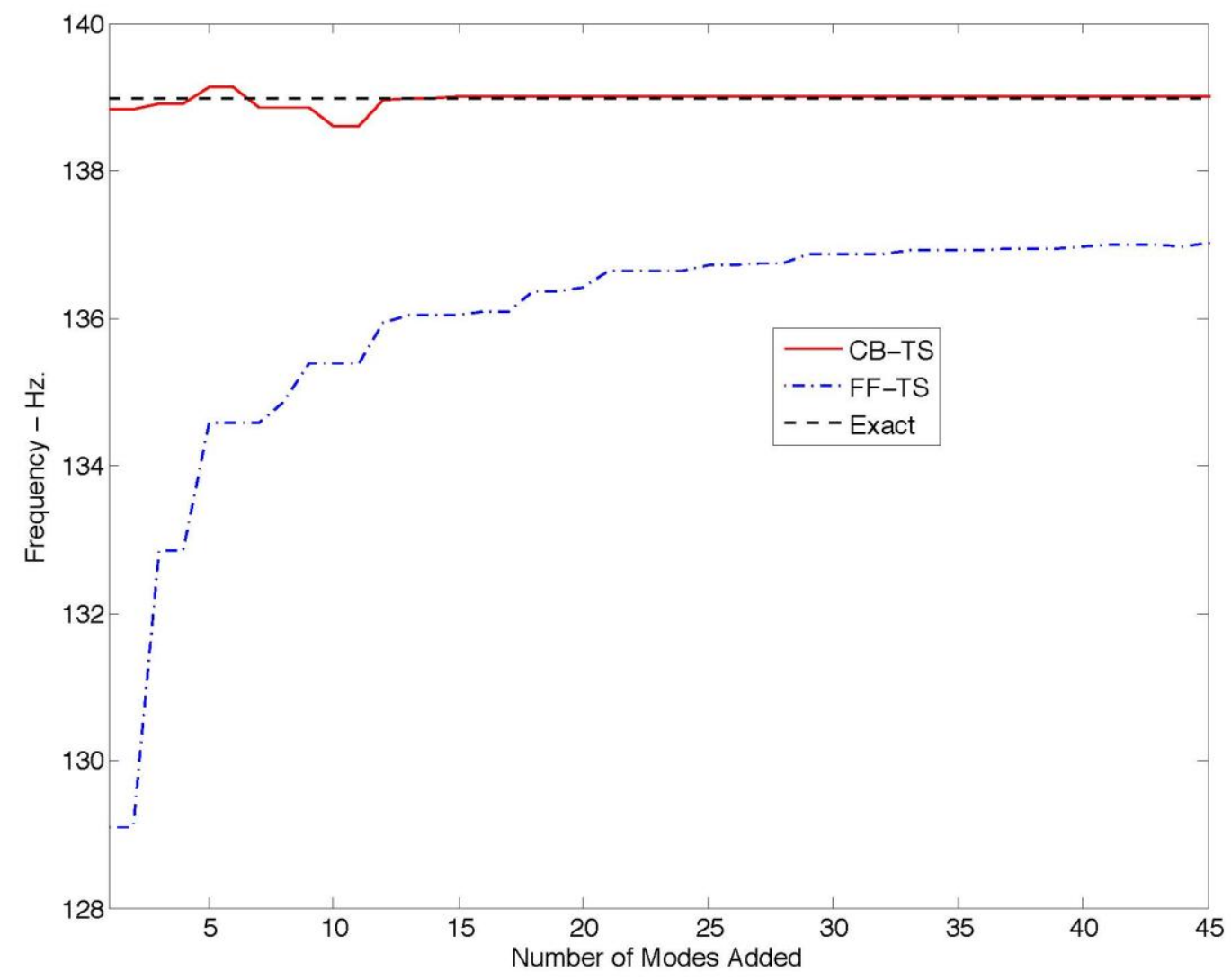

Fig. 9. Convergence of fundamental elastic synthesized system mode for CB-TS and FF-TS.

Table 2. System $E$ modal parameter comparison for perturbed TS.

$\begin{array}{rrrrr}\frac{\text { Mode }}{4.00} & \frac{\text { Exact }}{138.99} & \underline{\text { Predicted }} & \text { Error \% } & \text { CGM } \\ 5.00 & 207.87 & 211.22 & 1.41 & 1.00 \\ 6.00 & 229.35 & 229.49 & 0.06 & 1.00 \\ 7.00 & 414.35 & 414.03 & -0.08 & 1.00 \\ 8.00 & 456.82 & 459.35 & 0.55 & 1.00 \\ 9.00 & 489.64 & 494.30 & 0.95 & 1.00 \\ 10.00 & 985.64 & 994.79 & 0.93 & 1.00 \\ 11.00 & 1146.41 & 1162.96 & 1.44 & 0.99 \\ 12.00 & 1179.27 & 1184.01 & 0.40 & 0.99 \\ 13.00 & 1355.92 & 1371.25 & 1.13 & 1.00 \\ 14.00 & 1864.29 & 1876.94 & 0.68 & 1.00 \\ 15.00 & 1981.94 & 1990.33 & 0.42 & 1.00 \\ 16.00 & 2411.89 & 2416.67 & 0.20 & 1.00\end{array}$




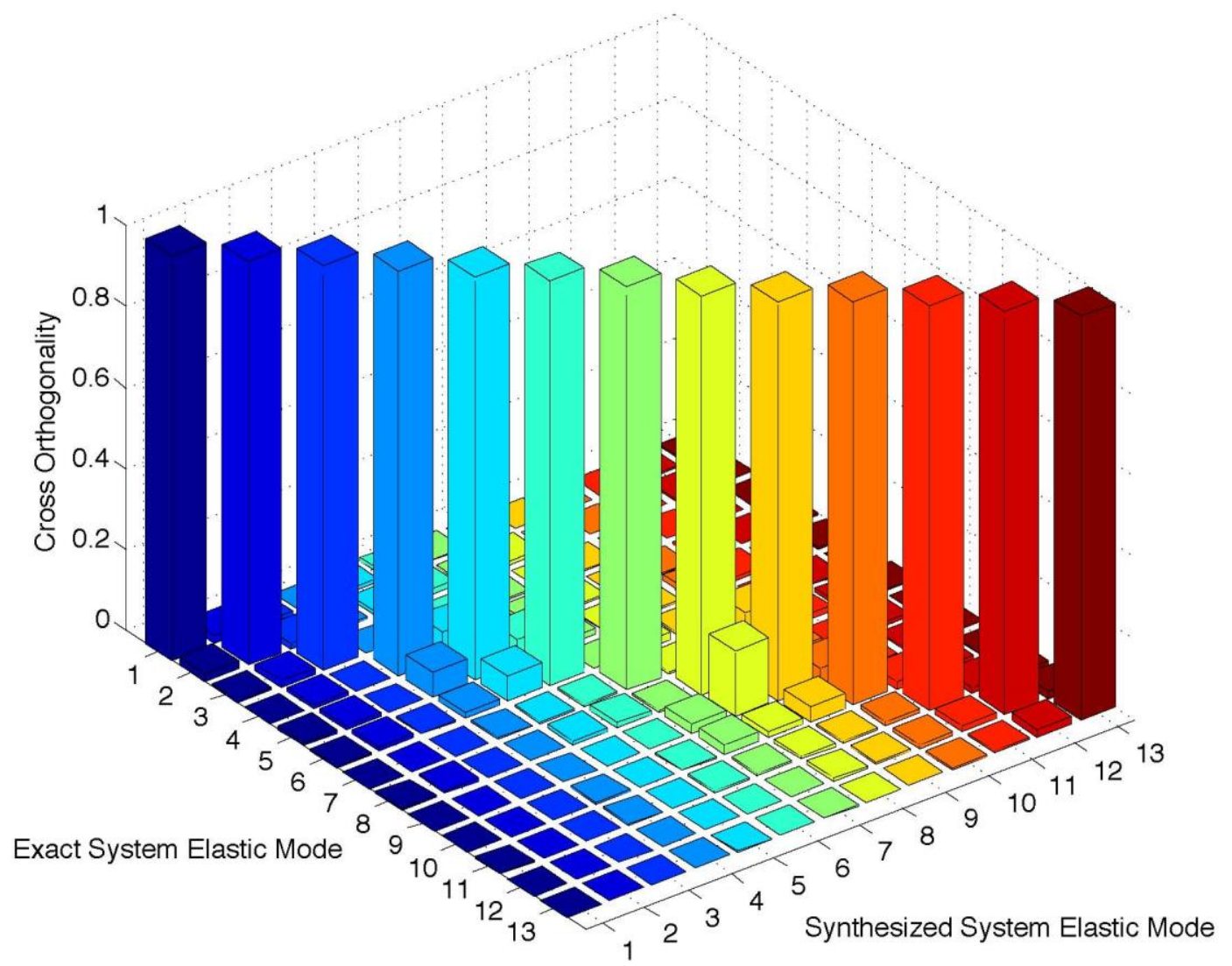

Fig. 10. System cross-orthogonality for first 13 elastic modes for perturbed TS. 


\section{Figure Captions:}

Fig. 1. Subtraction of TS from experimental substructure.

Fig. 2. Schematic of two-dimensional assembly $C$ with a statically indeterminate interface.

Fig. 3. Full assembly, $E=B+D$.

Fig. 4. Finite element mesh of the assembly of interest, $E$.

Fig. 5. Component $C$ elastic modes 5 (a) through 16 (1).

Fig. 6. Percentage error in satisfaction of constraints for free- and fixed-interface component $B$.

Fig. 7. Exact elastic mode shapes 4 (a) through 15 (1) for system $E$ FEM representation.

Fig. 8. System cross-orthogonality for first 15 elastic modes

Fig. 9. Convergence of fundamental elastic synthesized system mode for CB-TS and FF-TS

Fig. 10. System cross-orthogonality for first 13 elastic modes for perturbed TS. 


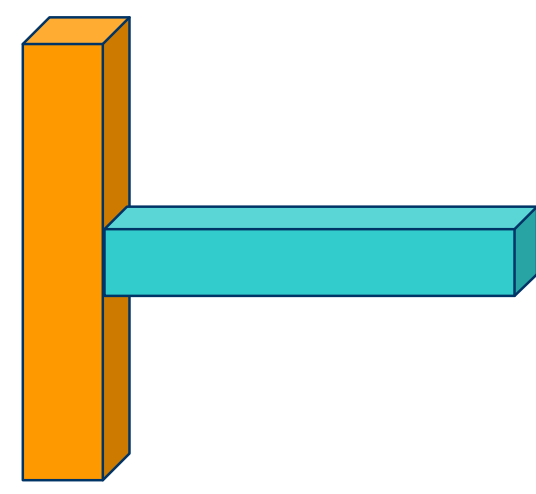

- =

Experimental Component: C
FEM Transmission Simulator: A
Experimental Based Component: B 


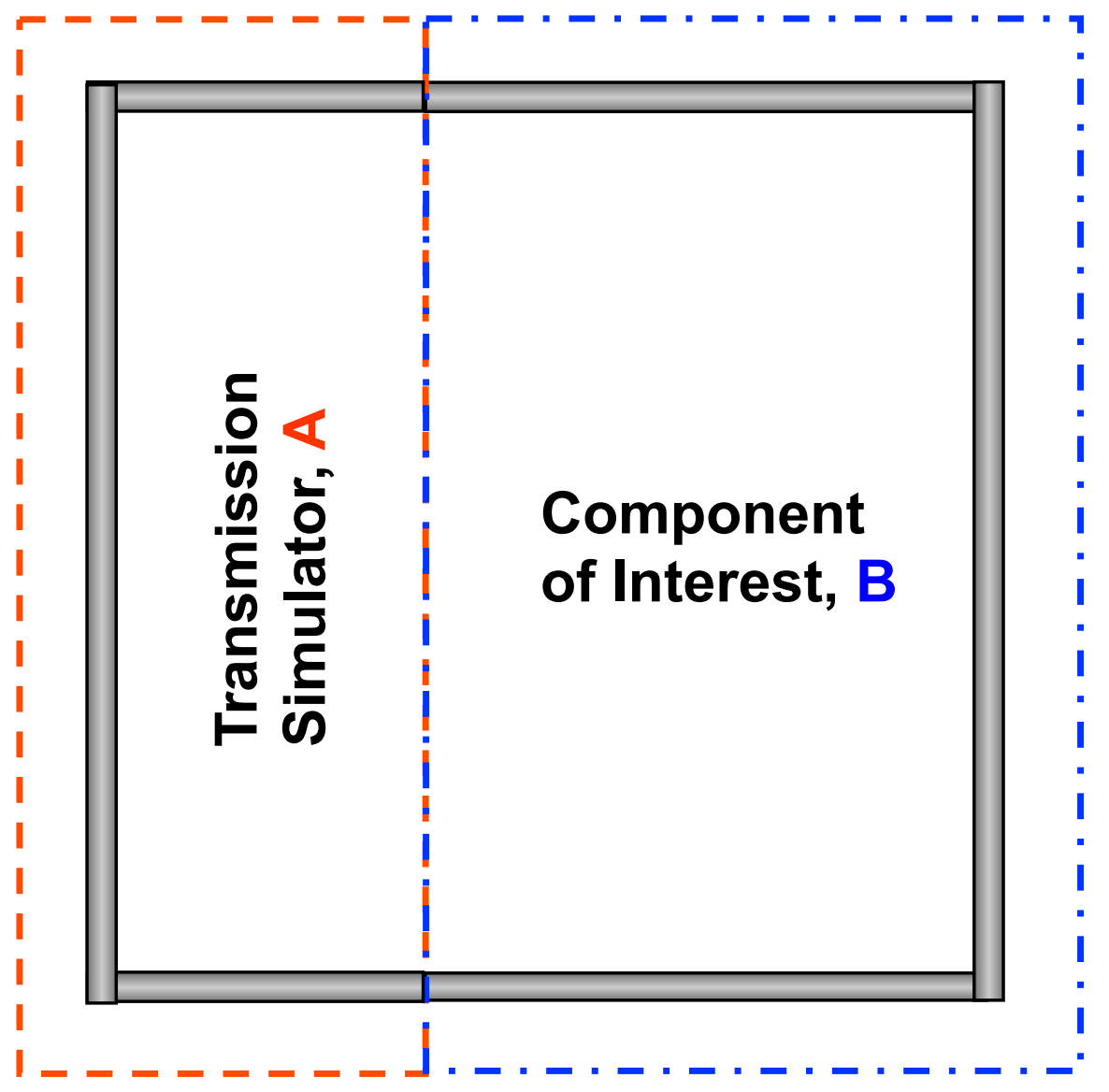




\section{Substructure,}

\section{Component of Interest, B}

D

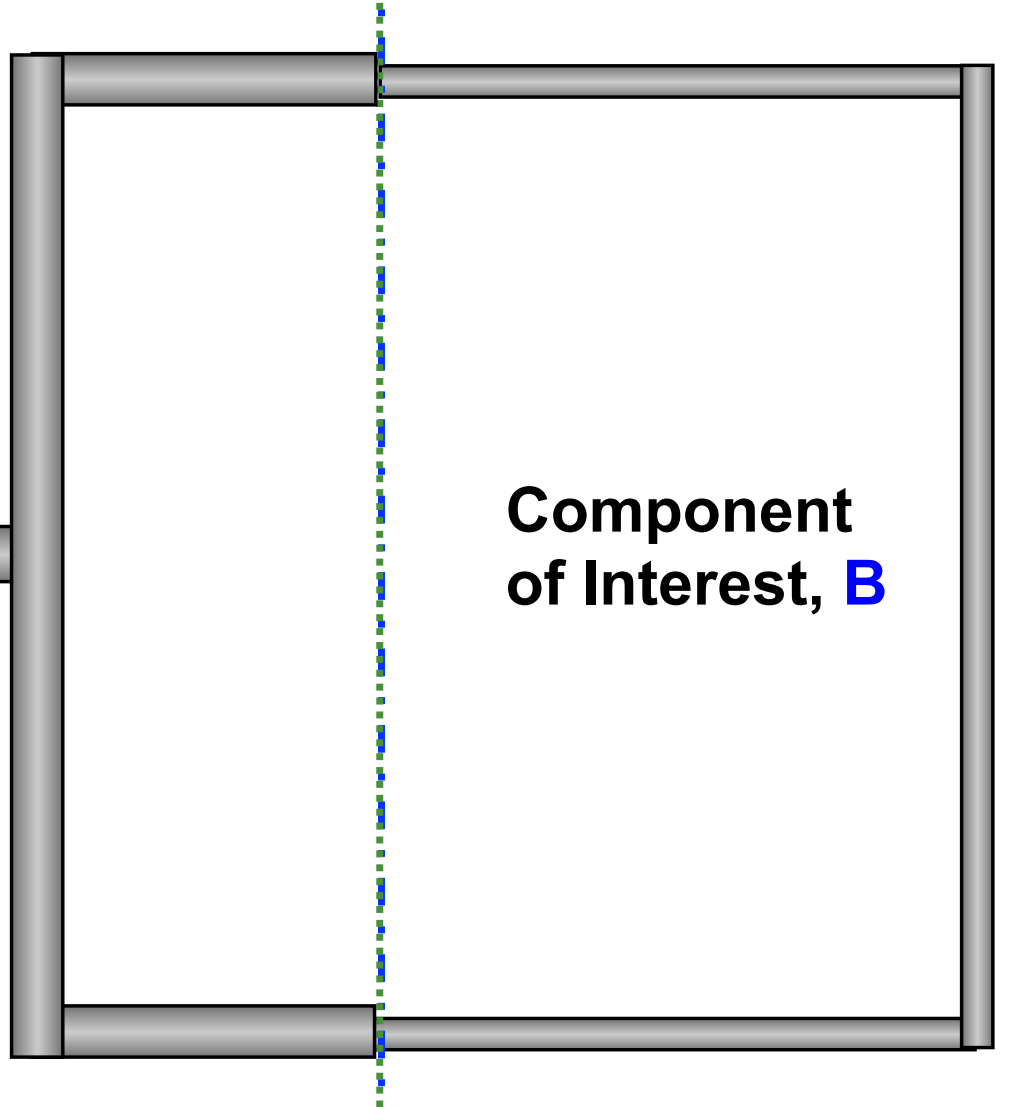




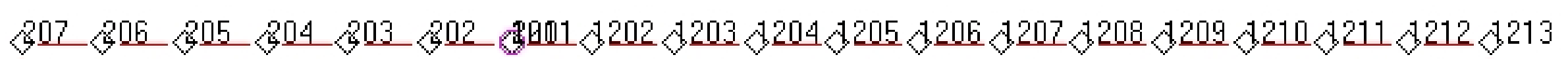

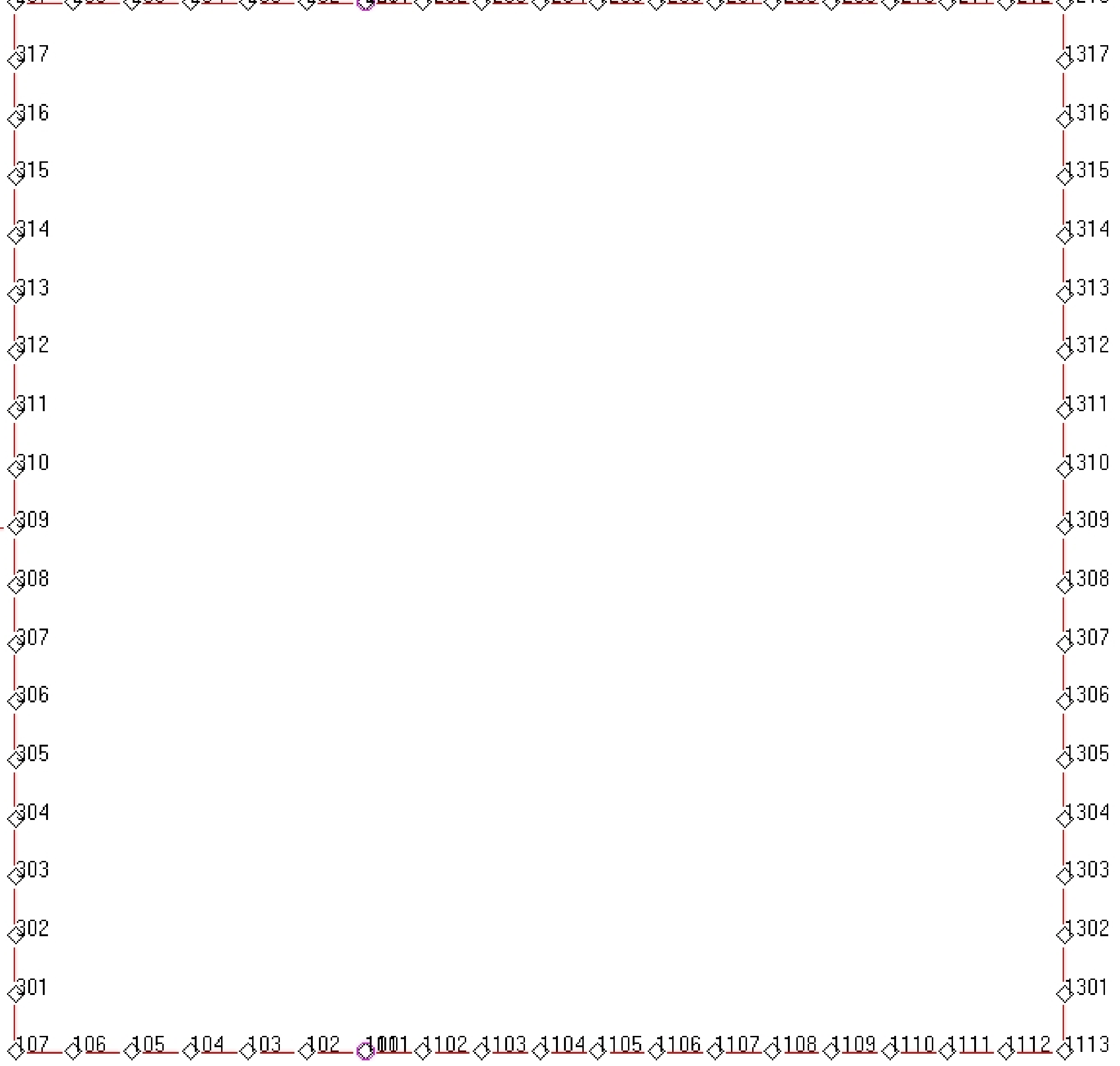




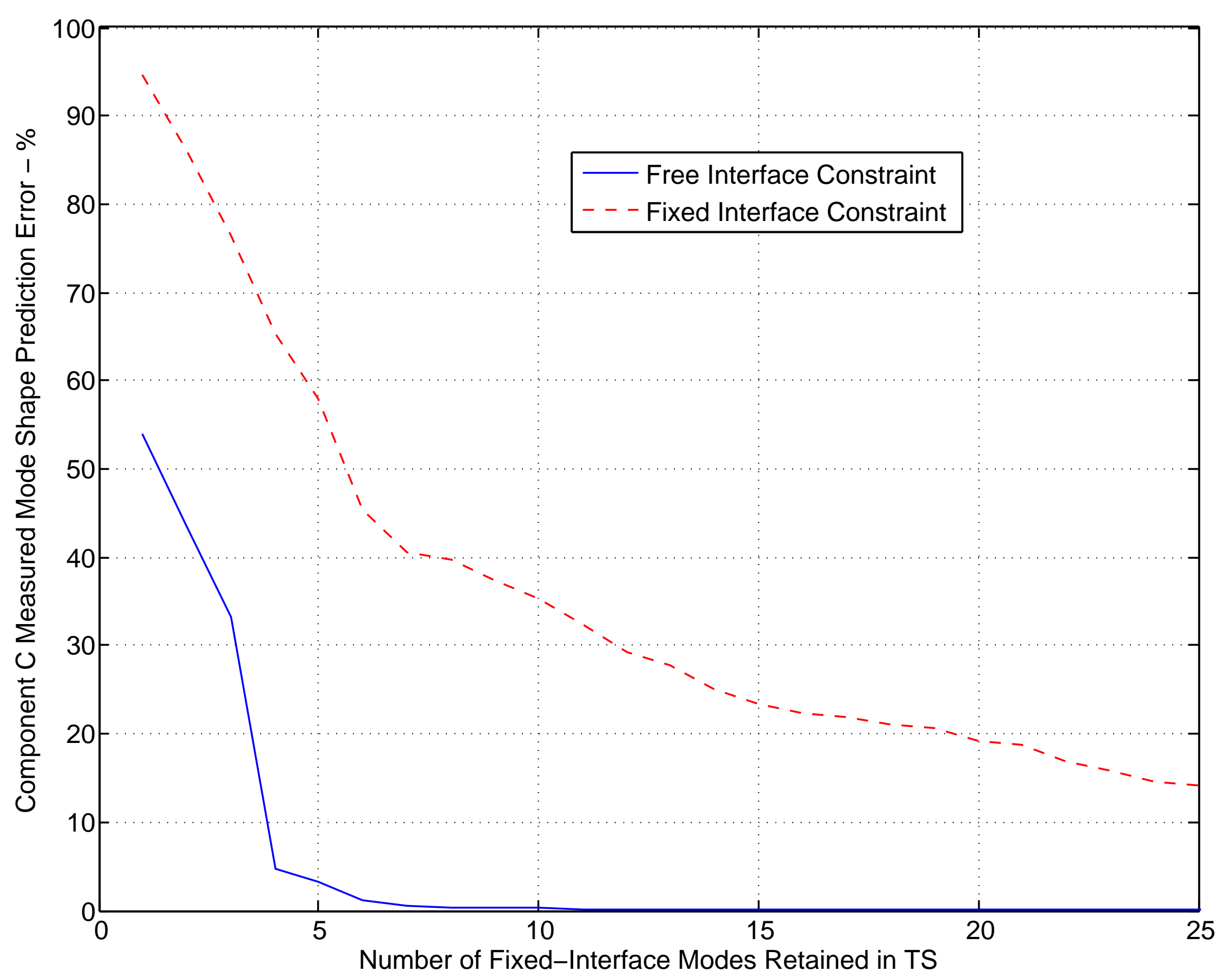




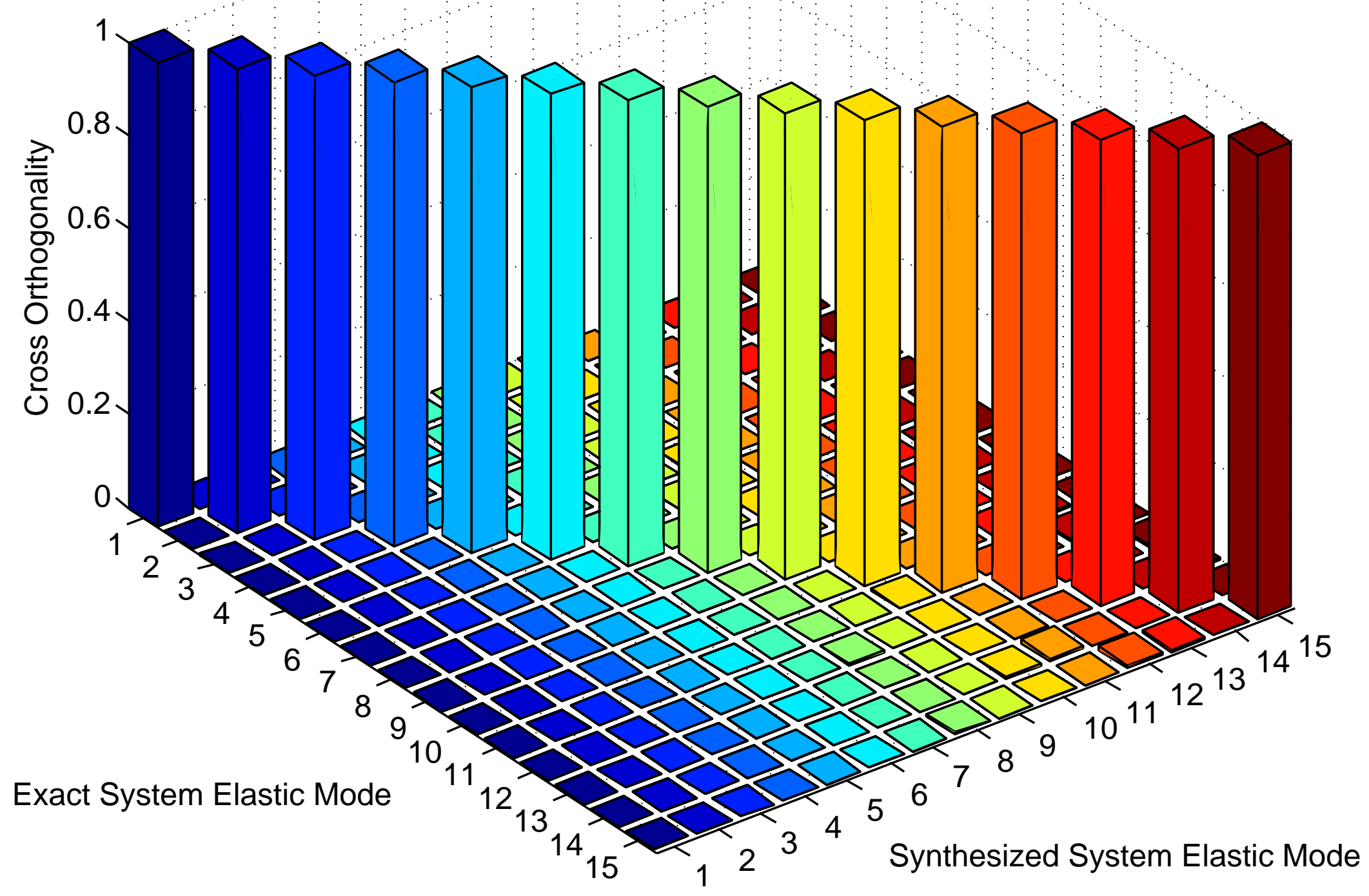




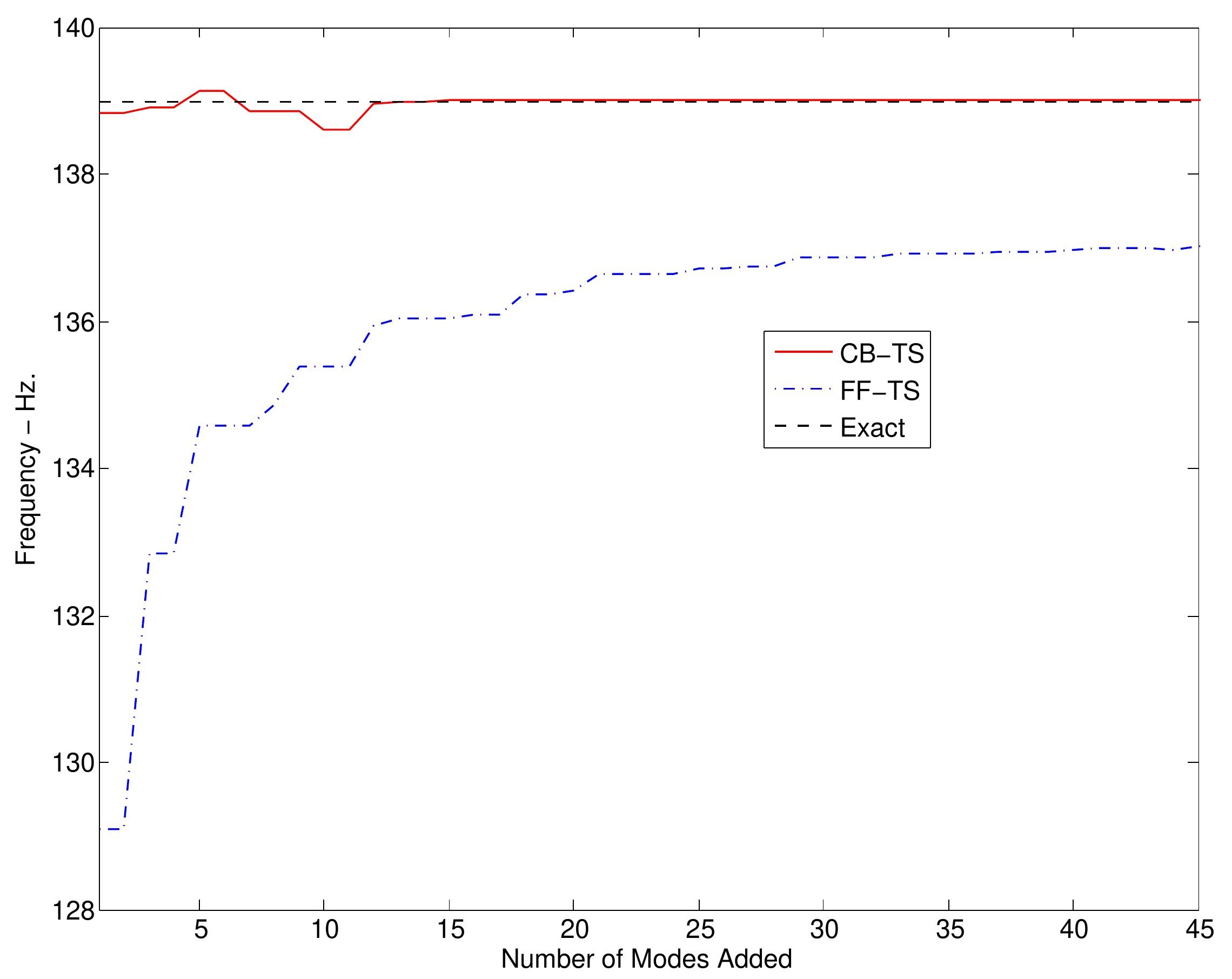




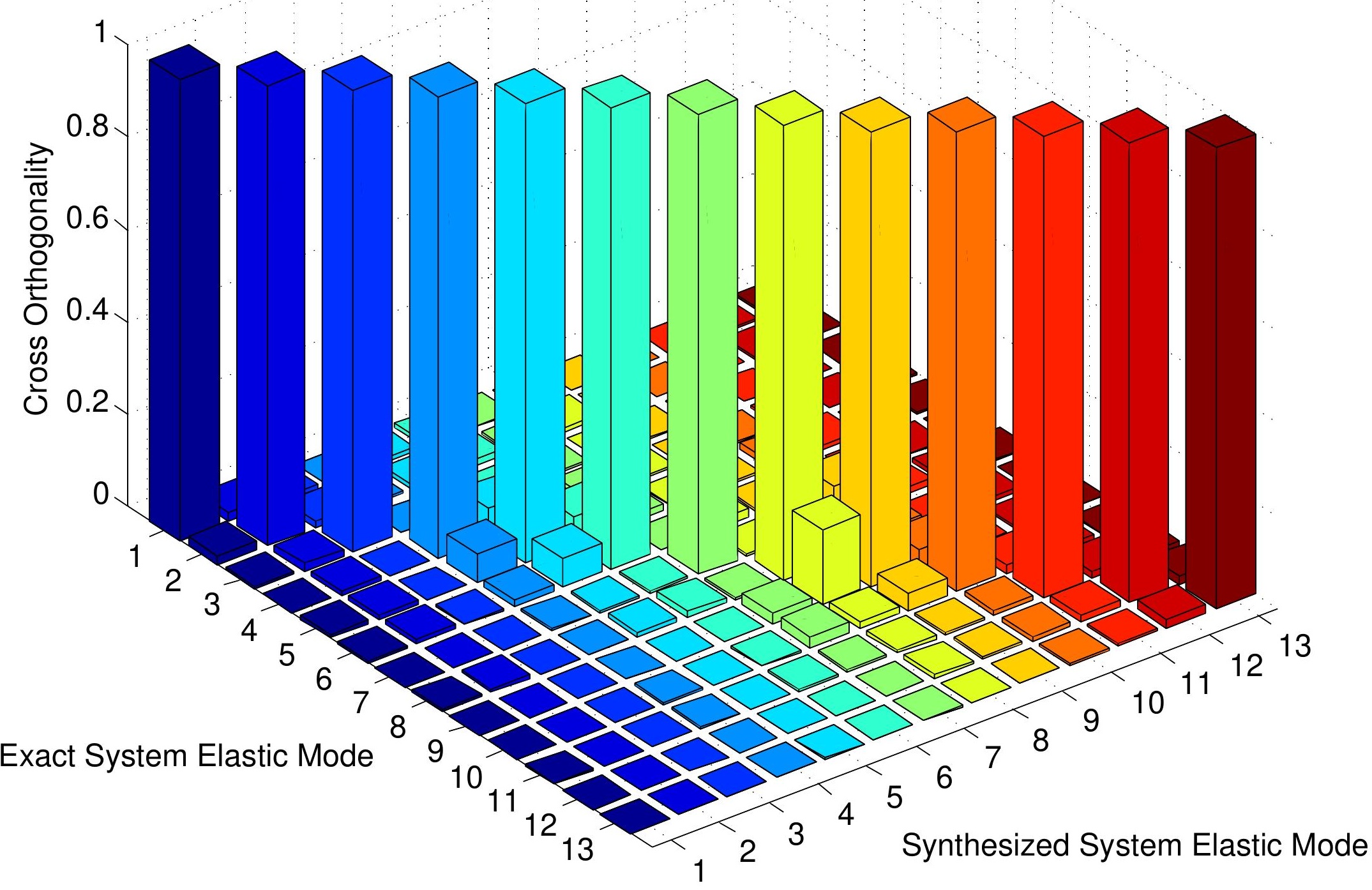

